\title{
The Cotton Lignin Biosynthetic Gene Gh4CL30 Regulates Lignification and Phenolic Content and Contributes to Verticillium Wilt Resistance
}

\author{
Xian-Peng Xiong, ${ }^{1}$ Shi-Chao Sun, ${ }^{1}$ Qian-Hao Zhu, ${ }^{2}$ Xin-Yu Zhang, ${ }^{1}$ Yan-Jun Li, ${ }^{1}$ Feng Liu, ${ }^{1}$ Fei Xue,,${ }^{1,+}$ \\ and Jie Sun ${ }^{1, \dagger}$ \\ ${ }^{1}$ Key Laboratory of Oasis Eco-agriculture, College of Agriculture, Shihezi University, Shihezi 832003, Xinjiang, China \\ ${ }^{2}$ CSIRO Agriculture and Food, GPO Box 1700, Canberra 2601, Australia
}

Accepted 30 October 2020.

\begin{abstract}
Verticillium wilt is a vascular disease causing tremendous damage to cotton production worldwide. However, our knowledge of the mechanisms of cotton resistance or susceptibility to this disease is very limited. In this study, we compared the defense transcriptomes of cotton (Gossypium hirsutum) cultivars Shidalukang 1 (Verticillium dahliae resistant, HR) and Junmian 1 ( $V$. dahliae susceptible, HS) before and after $V$. dahliae infection, identified hub genes of the network associated with responses to $V$. dahliae infection, and functionally characterized one of the hub genes involved in biosynthesis of lignin and phenolics. We identified 6,831 differentially expressed genes (DEGs) between the basal transcriptomes of HR and HS; 3,685 and 3,239 of these DEGs were induced in HR and HS, respectively, at different time points after $V$. dahliae infection. KEGG pathway analysis indicated that DEGs were enriched for genes involved in lignin biosynthesis. In all, 23 hub genes were identified based on a weighted gene coexpression network analysis of the 6,831 DEGs and their expression profiles at different time points after $V$. dahliae infection. Knockdown of $G h 4 C L 30$, one of the hub genes related to the lignin biosynthesis pathway, by virusinduced gene silencing, led to a decreased content of flavonoids, lignin, and $S$ monomer but an increased content of $\mathbf{G}$ monomer, G/S lignin monomer, caffeic acid, and ferulic acid, and enhanced cotton resistance to $V$. dahliae. These results suggest that Gh4CL30 is a key gene modulating the outputs of different branches of the lignin biosynthesis pathway, and provide new insights into cotton resistance to $\mathrm{V}$. dahliae.
\end{abstract}

\section{X.-P. Xiong and S.-C. Sun contributed equally to this work. \\ ${ }^{\dagger}$ Corresponding authors: J. Sun; sunjie@ shzu.edu.cn and F. Xue; xuefei@shzu.edu.cn}

Funding: This work was supported by the National Key Research and Development Program of China (grant number 2016YFD0100200), the National Natural Science Foundation of China (grant number 31960438), and the Genetically Modified Organisms Breeding Major Project of China (grant number 2016ZX08005-005).

*The $\boldsymbol{e}$-Xtra logo stands for "electronic extra" and indicates that supplementary figures and supplementary tables are published online.

The author(s) declare no conflict of interest.

(c) (1) $(9)$ Copyright $\odot 2021$ The Author(s). This is an open access article distributed under the CC BY-NC-ND 4.0 International license.
Keywords: caffeic acid, ferulic acid, Gh4CL30, Gossypium hirsutum, lignin, transcriptome, Verticillium wilt, weighted gene co-expression network analysis

Cotton (Gossypium hirsutum L.), one of the most important fiber crops in the world, has crucial economic and social relevance, especially in Xinjiang Province, which is now the main area of cotton cultivation in China (Tian et al. 2017). Verticillium wilt is caused by Verticillium dahliae, which is a vascular fungal pathogen that can infect over 200 plant species, including members of the Malvaceae family such as cotton. Verticillium wilt in cotton causes serious economic losses (Cai et al. 2009; Deketelaere et al. 2017; Fradin and Thomma 2006). V. dahliae enters roots through root tips or small natural wounds. It then rapidly spreads through the xylem vessels to the stem and leaves of the infected plant. After successful infection, $V$. dahliae secretes exopolysaccharide that likely affects the plant's ability to transport water and nutrients, resulting in blockage of xylem vessels, leaf wilting, and death (Klosterman et al. 2009).

Plants use both physical and biochemical defenses against pathogen infection (Glazebrook 2005). Phenylpropanoid metabolites such as lignins and phenolic compounds play an essential role in plant disease resistance (Boerjan et al. 2003; Vogt 2010). In plant cells, lignin is produced by the phenylalanine/ tyrosine metabolic pathway, one of the important branches of the phenylpropanoid pathway and part of a very complex metabolite network (Boerjan et al. 2003; Liu et al. 2018). Lignin formed by polymerization of monolignols provides a physical barrier to prevent entry and colonization of pathogen by reinforcing cell walls (Boerjan et al. 2003; Wang and Balint-Kurti 2016). Multiple enzymes are involved in lignin biosynthesis, including phenylalanine ammonia lyase (PAL), cinnamate 4-hydroxylase $(\mathrm{C} 4 \mathrm{H})$, 4-coumarate-CoA ligase (4CL), hydroxycinnamoyl transferase (HCT), $p$-coumarate 3 hydroxylase $(\mathrm{C} 3 \mathrm{H})$, caffeic acid O-methyltransferase (COMT), Ferulate 5-hydroxylase (F5H), caffeoyl shikimate esterase (CSE), caffeoyl CoA O-methyltransferase (CCoAOMT), cinnamoyl-CoA reductase (CCR), and cinnayl alcohol dehydrogenase (CAD) (Boerjan et al. 2003; Vanholme et al. 2010).

Suppressing the expression of lignin biosynthesis-related genes such as COMT1 and CAD in Arabidopsis and PALA in rice enhanced plant susceptibility to pathogens (Tonnessen et al. 2015; Tronchet et al. 2010). Wheat plants with silenced TmPAL, TmCOMT, TmCCoAMT, or TmCAD decreased resistance to Blumeria graminis f. sp. tritici. Cosilencing two of these 
genes $(T m P A L+T m C A O M T, T m P A L+T m C A D$, or TmCAOMT+ $T m C A D)$ further enhanced disease susceptibility compared with silencing individual genes (Bhuiyan et al. 2009). Lignin biosynthesis-related genes have also been linked to defense against diseases in alfalfa, tobacco, potato, and cotton (Gill et al. 2018; Hu et al. 2019; Shadle et al. 2003; Xu et al. 2011). $4 \mathrm{CL}$ is the third key enzyme in the phenylpropanoid metabolic pathway which contributes to biosynthesis of lignin and flavonoids (Vogt 2010). Previous studies have shown that the expression level of $4 C L$ is strongly induced by pathogens in different plants (Oliveira et al. 2015; Sun et al. 2013; Xu et al. 2011). However, except for a $4 C L$-like gene, OsAAE3 (Liu et al. 2017), the function of $4 C L$ in plant disease resistance is rarely reported.

Inhibition of steps of the phenylpropanoid pathway may lead to accumulation or reduction of phenolic compounds (Konig et al. 2014). Phenolic compounds generated in the various steps of the phenylpropanoid pathway have a wide range of roles in plants, including growth, development, reproduction, adaptation, and protection from biotic and abiotic stresses (Lavhale et al. 2018; Mierziak et al. 2014). The function of phenolic compounds in plant-pathogen interactions is well known, by which they have strong antibacterial and antifungal qualities (Lanoue et al. 2010). Some reports have provided evidence to suggest that phenolic compounds play important defense roles in cotton's response to V. dahliae infection, whereas only a few specific monophenols have been functionally characterized (Gao et al. 2013; Y. Zhang et al. 2017).

$G$. hirsutum and $G$. barbadense are the two major allotetraploid cotton species, contributing more than $90 \%$ of worldwide fiber production (Wang et al. 2019). Although both G. hirsutum and $G$. barbadense originated from one hybridization event between the ancestor of $G$. herbaceum (A1) or G. arboreum (A2) and the ancestor of $G$. raimondii (D5) approximately 1 million to 1.5 million years ago, they have great differences in fiber quality, disease resistance, production, and other traits (Wendel 1989). G. barbadense varieties often show high resistance to $V$. dahliae but few $G$. hirsutum varieties do (Zhang et al. 2013). A number of RNA sequencing (RNA-seq) experiments have been carried out in cotton to investigate the defense transcriptome against $V$. dahliae and the mechanism of cotton- $V$. dahliae interaction (Zhang et al. 2013). Most investigations had a focus on resistant $G$. barbadense accessions (Xu et al. 2011, 2014; Zhang et al. 2013) or compared the differences between resistant $G$. barbadense accessions and susceptible G. hirsutum accessions (Gao et al. 2013; Sun et al. 2013), although one study compared the defense transcriptomes of $V$. dahliaeresistant and $V$. dahliae-susceptible $G$. hirsutum varieties (W. Zhang et al. 2017). The mechanisms of the molecular interactions that confer high levels of resistance versus susceptibility in the cotton- $V$. dahliae interaction remain to be fully elucidated.

In this study, we compared the basal and defense transcriptomes of a $V$. dahliae-resistant (HR) and a $V$. dahliae-susceptible (HS) cotton ( $G$. hirsutum) variety, and identified enriched Kyoto Encyclopedia of Genes and Genomes (KEGG) pathways and hub genes associated with cotton response to $V$. dahliae infection. Functional characterization demonstrated that silencing Gh4CL30, one of the hub genes involved in the lignin biosynthesis pathway, was able to enhance $V$. dahliae resistance, which could be achieved by increasing the ratio of the G/S lignin monomer and the contents of caffeic acid and ferulic acid.

\section{RESULTS}

\section{Characterization of the two cotton cultivars in response} to $V$. dahliae infection.

We initially conducted disease assays to confirm the level of $V$. dahliae response of the two G. hirsutum cultivars (Shidalukang
1: HR and Junmian 1: HS) to be used. At 21 days postinfection (dpi), we observed significant difference of disease response between HR and HS. All HS seedlings exhibited strong disease symptoms and ultimately died, whereas the majority of HR seedlings showed no obvious disease symptoms (Fig. 1A). Both the ratio of diseased plants and disease index of HS were significantly higher than those of HR at 14 and 21 dpi (Fig. 1B and C). Moreover, fungal recovery assays, vascular browning observation, and fungal biomass analysis all indicated that the level of $V$. dahliae resistance of HR was much higher than that of HS (Fig. 1D to F).

\section{HR and HS showed different basal transcriptome.}

We performed RNA-seq using samples from $0,12,24$, and $48 \mathrm{~h}$ postinoculation (hpi) from HR and HS. In total, 1.20 billion clean reads (approximately $180.35 \mathrm{~Gb}$ of data) were obtained (Supplementary Table S2). Across all samples, the percentage of nucleotides with a quality score above 20 was over $97.67 \%$, and the GC percentage ranged from 43.03 to $43.69 \%$ (Supplementary Table S2). Approximately 95\% of the clean reads were mapped to the G. hirsutum reference genome (TM-1) (Zhang et al. 2015). Of the approximately 5\% clean reads that did not align to the TM-1 genome, only 2.53 to $4.63 \%$ could be aligned to the $V$. dahliae genome (Klosterman et al. 2011) (Supplementary Table S3).

To investigate whether the basal transcriptomes of HR and HS are related to their different disease response, we compared the 0-hpi transcriptomes (i.e., uninfected by V. dahliae) of HR and HS and identified 6,831 differentially expressed genes (DEGs) between HR and HS using DESeq (adjusted $P$ value $<0.05$ ). Of these DEGs, 2,866 (type I) and 3,965 (type II) had significantly lower and higher expression levels, respectively, in HS than in HR (Fig. 2A; Supplementary Tables S4 and S5). We selected six type I DEGs (HR > HS) for confirmation using reverse-transcription quantitative PCR (RTqPCR). As expected, the expression levels of all six genes (GhABC1, GhABC2, GhMLP1, GhMLP2, GhWAK1, and GhRPS1) in the roots or stems were higher in HR than in HS (Supplementary Fig. S1A and B), suggesting the reliability of the RNAseq results.

To predict the functions of the two types of DEGs, KEGG pathway analysis was performed for each type of DEG. The result showed that the type I DEGs enriched for only six KEGG pathways $(P$ value $<0.05)$ (Fig. 2B; Supplementary Table S6), including DNA replication, carbon fixation in photosynthetic, pyrimidine metabolism, pentose phosphate pathway, mismatch repair, and flavonoid biosynthesis. For type II DEGs, 11 KEGG pathways were found to be enriched (Fig. 2B; Supplementary Table S7). Consistent with other reports, some of these enriched pathways were associated with plant responses to $V$. dahliae infection; for example, flavonoid biosynthesis and phenylpropanoid biosynthesis (Guo et al. 2017; Xu et al. 2011). Genes related to the flavonoid biosynthesis pathway were enriched in both HR and HS; however, the phenylpropanoid biosynthesis pathway was enriched only in HS (Fig. 2B).

\section{Genes response to stimulus and stresses were highly expressed in HR.}

To further explore the functions of DEGs, the two types of DEGs were annotated based on functional gene ontology (GO) using the default Blast2GO parameters. Of the top $20 \mathrm{GO}$ terms $(P$ value $<0.05)$, none overlapped between the type I and type II DEGs (Fig. 2C; Supplementary Tables S8 and S9). Overall, processes involved in DNA binding (GO:0003677) and response to stimulus (GO:0050896) were the most enriched in type I DEGs (Fig. 2C). Interestingly, in total, 292 genes responsive to stimulus (GO:0050896; $P=0.013$ ) and 148 genes 
associated with stress responses (GO:0006950; $P=0.005)$ were discovered in HR but not in HS (Fig. 2C), including five ABC transporters, two major latex proteins (MLPs), one wallassociated receptor-like kinase, and several disease resistance proteins (nucleotide binding leucine-rich repeat protein and nucleotide binding adaptor shared by APAF-1, resistance proteins, and CED-4 domain protein). In type II DEGs, the two major GO terms were metabolic process (GO:0008152) and catalytic activity (GO:0003824) (Fig. 2C).

Identification of DEGs in response to $V$. dahliae infection.

To examine the changes of the basal transcriptome of HR and HS in response to $V$. dahliae infection, we performed pairwise transcriptome comparisons between the infected $(12,24$, and 48 hpi) and uninfected (0 hpi) HR and HS. In HR, 5,146, 8,011, and 9,014 DEGs were identified at 12, 24, and 48 hpi, respectively (Supplementary Fig. S2A). The numbers of DEGs identified at 12, 24, and $48 \mathrm{hpi}$ in HS were 6,970, 7,754, and 6,392, respectively (Supplementary Fig. S2B). In total, 13,250 and 12,602 nonredundant DEGs were identified in HR and HS, respectively, and 5,930 were common in HR and HS (Supplementary Fig. S2C).

Of the type I and II basal DEGs identified between HR and HS, 782 and 943, respectively, were not significantly changed in response to $V$. dahliae infection. Of the type I DEGs responsive to $V$. dahliae infection, 903 and 461 responded in only HR and only HS, respectively, and 720 responded in both HR and HS (Fig. 2D). Likewise, following $V$. dahliae infection, 946 and 960 type II DEGs responded in only HR and only HS, respectively, and 1,098 responded in both HR and HS (Fig. 2D). Of the total 6,831 type I and II DEGs, 3,685 and 3,239 responded to $V$. dahliae infection in HR and HS, respectively (Fig. 2D; Supplementary Tables S10 and S11). These genes were mapped to 108 and 114 KEGG pathways, with 13 and 12 enriched pathways ( $P$ value $<0.05)$, respectively. Eight of them were enriched in both HR and HS (Fig. 2E; Supplementary Tables S12 and S13). Notably, the phenylpropanoid biosynthesis pathway was only enriched in HR.
A

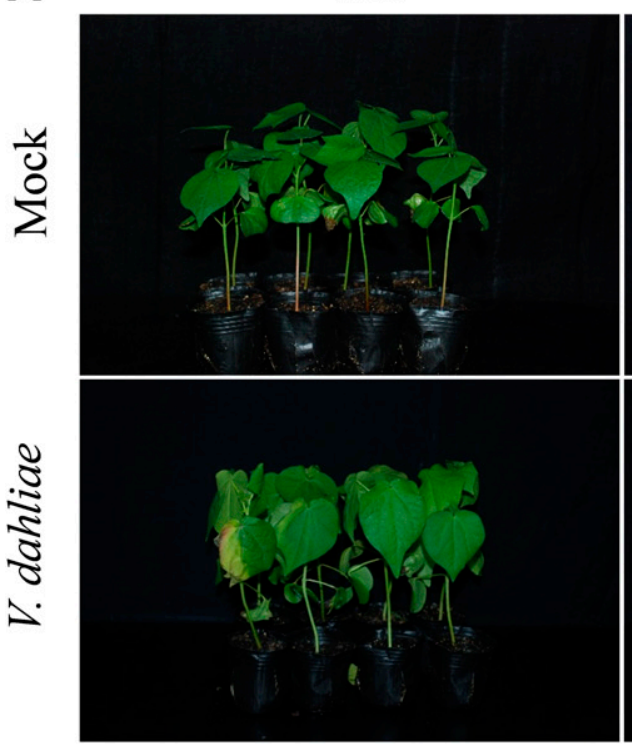

C

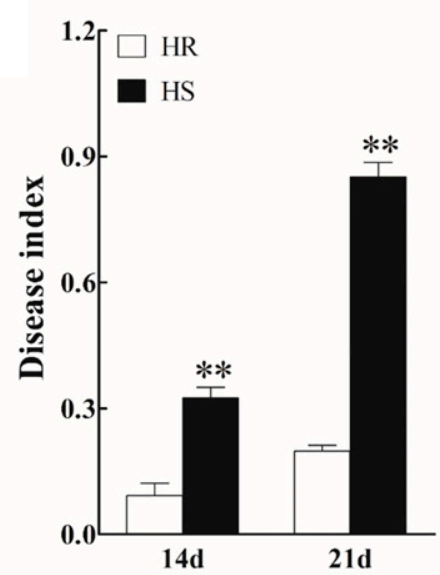

HS

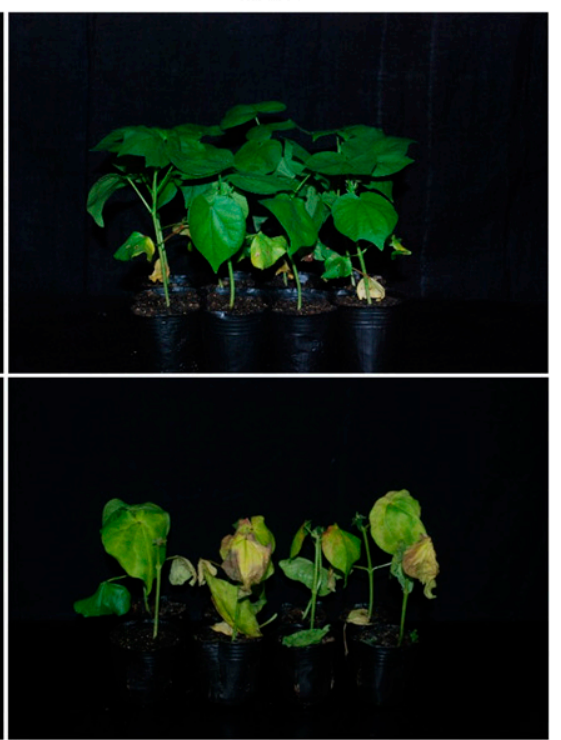

D

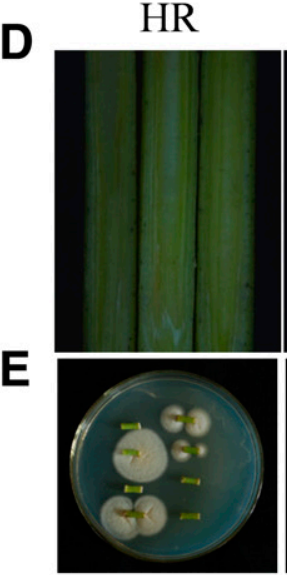

HS

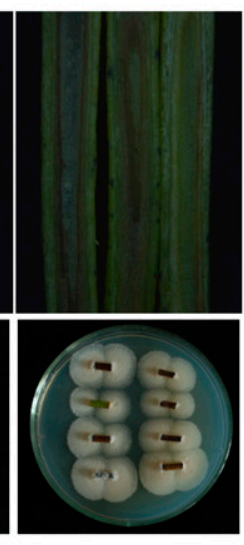

B
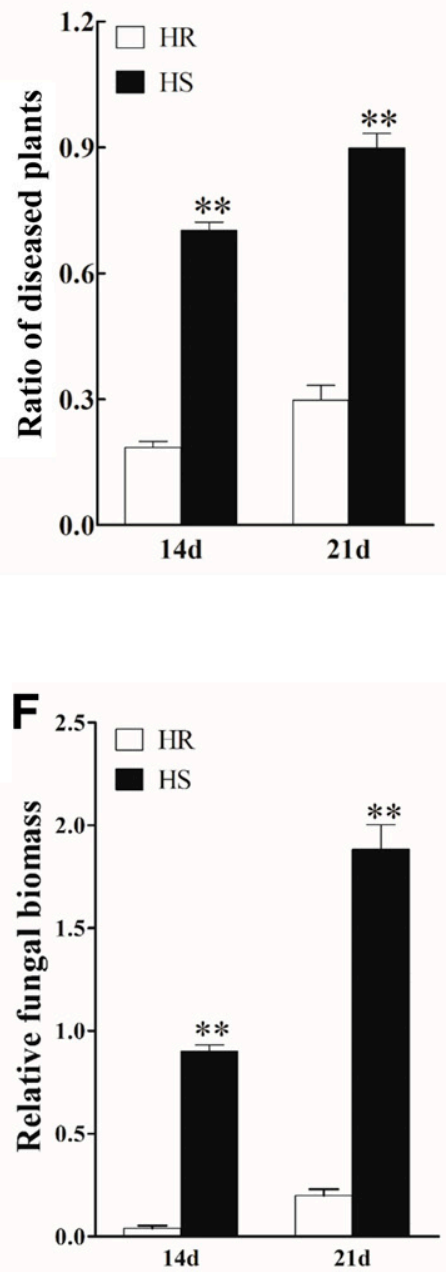

Fig. 1. Levels of resistance of resistant Shidalukang 1 (HR) and susceptible Junmian 1 (HS) to Verticillium dahliae. A, Phenotypes of HR and HS at 21 days postinfection (dpi) with $V$. dahliae. B and C, Ratio of diseased plants and disease index of HR and HS at 14 and 21 dpi. D, Comparison of disease symptoms in stems of HR and HS at 14 dpi. E, Fungal isolation in the stem sections from HR and HS at 14 dpi. Stems were plated on potato dextrose agar medium. Photos were taken after 7 days of culture at $25^{\circ} \mathrm{C}$. F, Quantitative PCR analysis of the relative fungal biomass in stems of HR and HS at 14 dpi. Data are the mean \pm standard error of three independent biological replicates, and asterisks indicate significant differences between groups determined using the Student's $t$ test (* and ** indicate $P<0.05$ and 0.01 , respectively). 
Lignin biosynthesis pathways were activated in both HR and HS cultivars after $V$. dahliae infection.

The phenylpropanoid metabolic pathway plays an important role in cotton defense against $V$. dahliae (Xu et al. 2011). Eleven classes of lignin biosynthesis-related genes were identified in the phenylpropanoid biosynthesis pathway. All of these genes were significantly induced in HR or HS upon $V$. dahliae infection, including six $G h P A L$, one $G h C 4 H$, four $G h 4 C L$, two GhHCT, two GhC3H, six GhCOMT, one $G h F 5 H$, two GhCSE, seven GhCCoAOMT, two GhCCR, and two GhCAD (Fig. 3A; Supplementary Table S14). Based on their expression profiles at 0 and 48 hpi in HR and HS, these genes could be clustered into two groups. The expression levels of the first group of genes were consistently higher in HS than in HR at both time points (Supplementary Fig. S3), while the expression levels of the second group of genes were higher in HS than in HR at 0 hpi but were variable between HR and HS at 48 hpi (with a similar expression level in most cases) (Supplementary Fig. S4).

When stained with phloroglucinol, the stem cross-sections of HS plants exhibited deeper red than HR at 0 and 48 hpi (Fig. 3B). Consistent with this observation, HS plants had a significantly higher level of lignin than HR plants at both 0 and 48 hpi (Fig. 3C). In both HS and HR plants, the lignin content was significantly higher at 48 hpi than at 0 hpi (Fig. $3 \mathrm{C})$. Of the two major lignin monomers, the contents of the $G$ and $\mathrm{S}$ monomers were significantly higher in $\mathrm{HS}$ than in $\mathrm{HR}$ at both 0 and 48 hpi, and also significantly increased in both HS and HR plants following $V$. dahliae infection (Fig. 3D, E). Compared with $0 \mathrm{hpi}$, the $\mathrm{G} / \mathrm{S}$ ratio was significantly increased and decreased in HR and HS plants, respectively, at 48 hpi (Fig. 3F).

\section{Weighted gene coexpression network analysis.}

To determine the potential role and the response of the basal transcriptome upon $V$. dahliae infection, we did weighted gene coexpression network analysis (WGCNA) analysis using the 6,831 DEGs identified between HR and HS at 0 hpi. These DEGs were grouped into 11 different modules based on pairwise correlation analysis of gene expression across all samples (Fig. 4). Five modules (Fig. 4B, indicated by a red underline) had a $P$ value $<0.01$ and a corresponding correlation coefficient $>0.5$ in at least one of the samples.

The purple module included 115 genes that were induced faster in HS than in HR (Supplementary Table S15). The expression levels of the 1,195 genes included in the brown module were quickly decreased in HS after $V$. dahliae infection, whereas the expression levels of the blue module genes were quickly reduced in HR after $V$. dahliae infection (Supplementary Table S15). The yellow module included 615 genes that were slightly decreased at 24 and 48 hpi in HS but increased in HR at 24 hpi (Supplementary Table S15). The expression levels of the green module genes were rapidly decreased at $12 \mathrm{hpi}$ in HS but increased in HS at 24 and 48 hpi (Supplementary Table S15). In total, 21 hub genes were identified based on the criteria of eigengene-based connectivities $>0.9$ and protein-protein interaction $>5$ (Table 1 ).

\section{Expression pattern of Gh4CL30.}

According to KEGG, WGCNA, and lignin content analyses, several genes of the lignin biosynthesis pathway are promising candidate genes related to the different $V$. dahliae resistance levels between HR and HS, including Gh_D10G0473, one of the hub genes identified based on gene network analysis. We have systematically analyzed the cotton $4 C L$ gene family and named Gh_D10G0473 as Gh4CL30 (Sun et al. 2020).
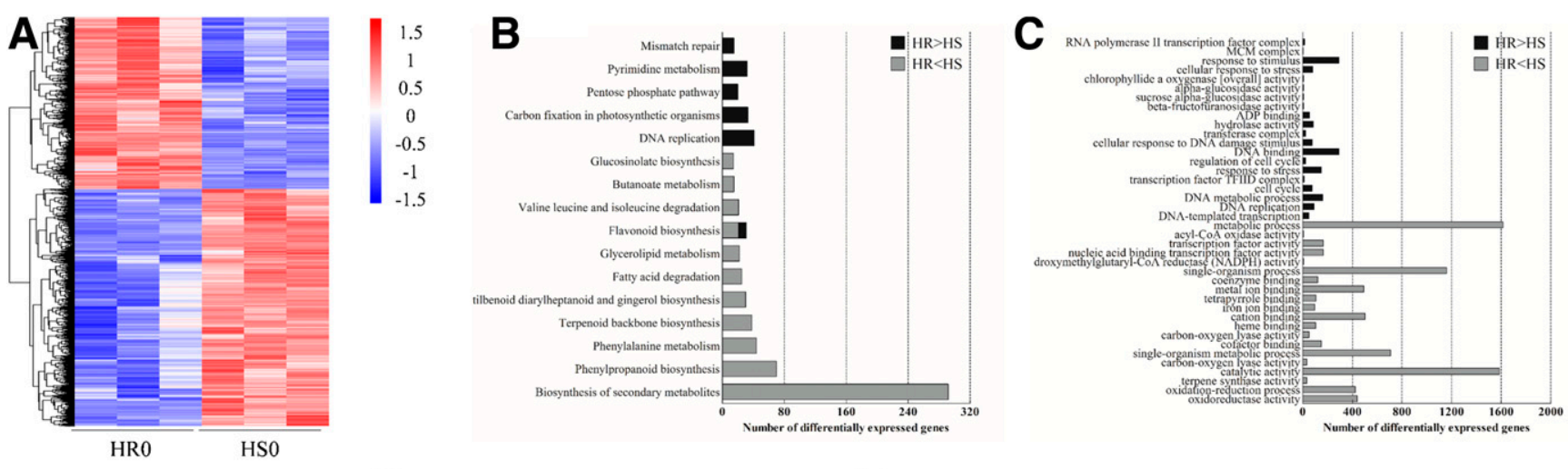

D
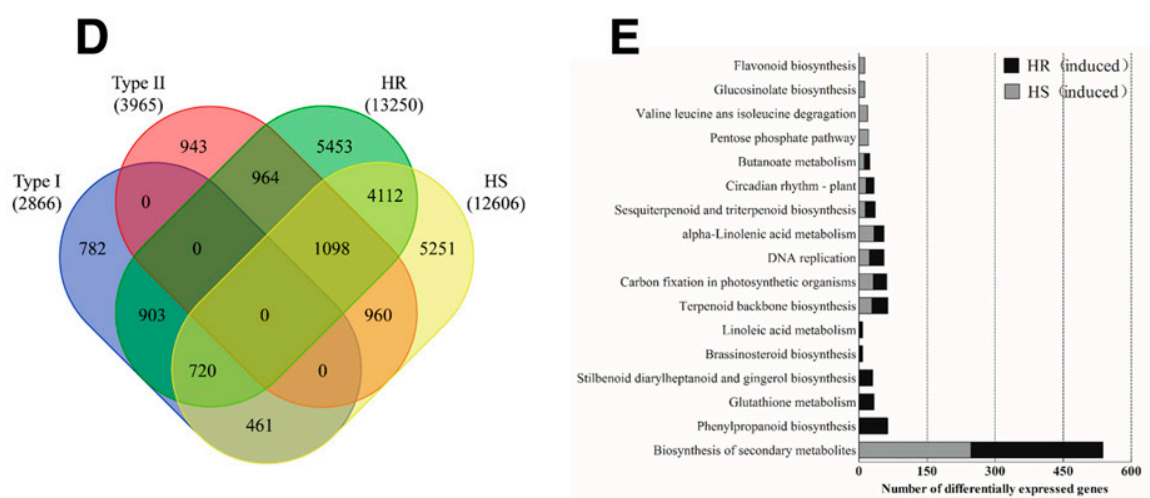

Fig. 2. Basal transcriptomes of resistant Shidalukang 1 (HR) and susceptible Junmian 1 (HS) (from Verticillium dahliae-uninfected plants) and their responses to $V$. dahliae infection. A, Heatmap of the 6,831 differentially expressed genes (DEGs) identified in the basal transcriptomes of HR and HS. B and C, Kyoto Encyclopedia of Genes and Genomes (KEGG) and gene ontology analyses of the 2,866 and 3,965 basal DEGs with a higher expression level in HR and HS, respectively. D, Venn diagram showing the number of the basal DEGs that were induced after $V$. dahliae infection in HR and HS. E, KEGG analysis of the basal DEGs that were significantly induced in HR and HS. 
A

Phenylalanine

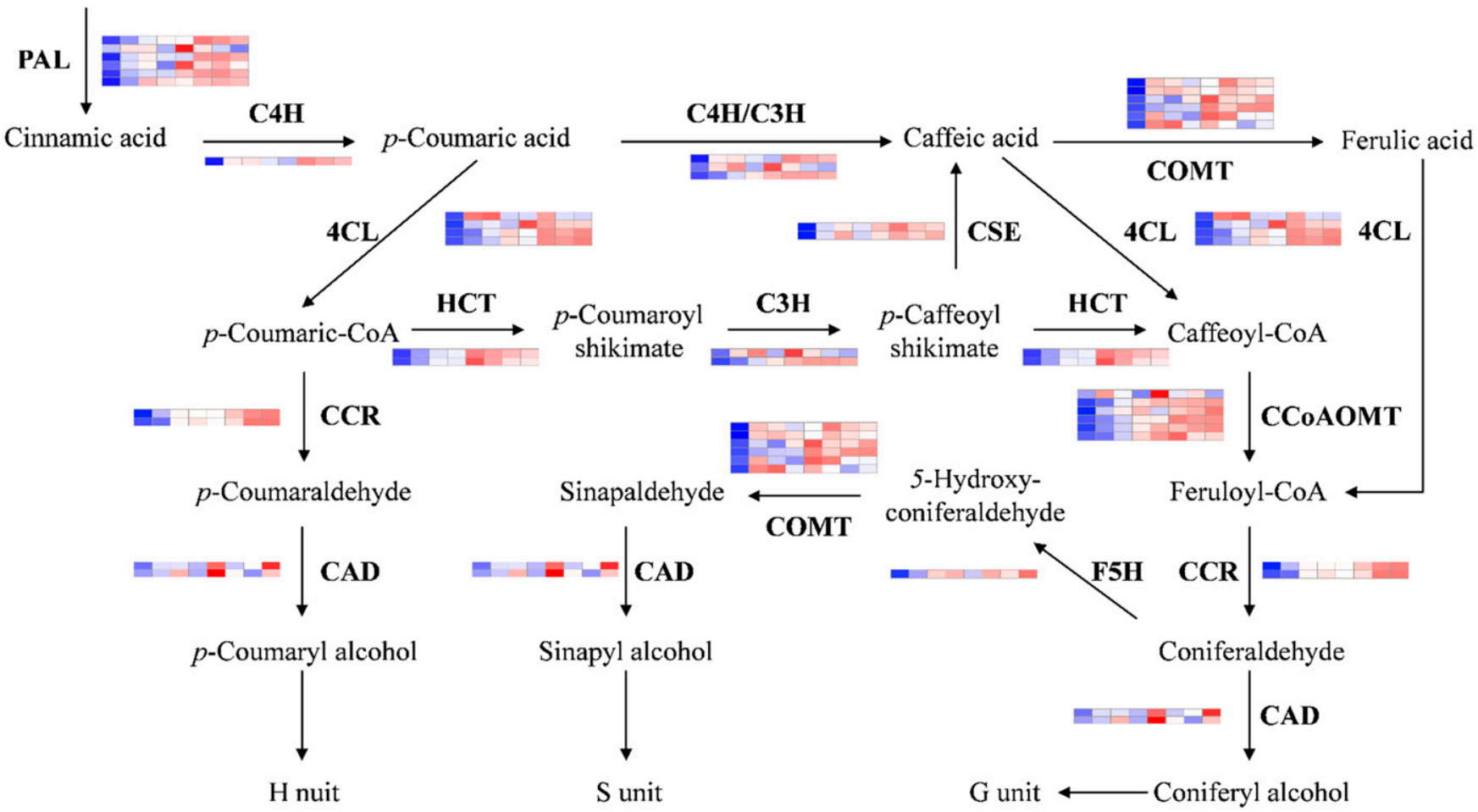

\begin{tabular}{|c|c|c|c|c|c|c|c|c|c|c|c|c|}
\hline \multirow[b]{2}{*}{ Left } & \multicolumn{8}{|c|}{ Sample order } & \multirow{3}{*}{ Right } & \multicolumn{3}{|c|}{ Normalized expression level } \\
\hline & HR0 & HR12 & HR24 & HR48 & HSO & HS12 & HS24 & HS48 & & & & \\
\hline & & & & & & & & & & 1.5 & 0 & -1.5 \\
\hline
\end{tabular}

B

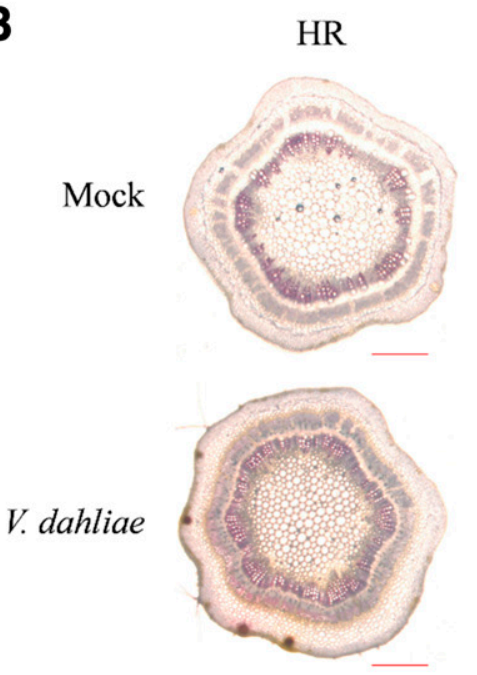

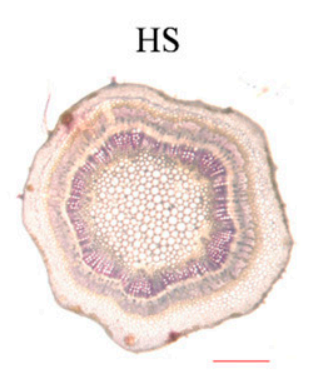

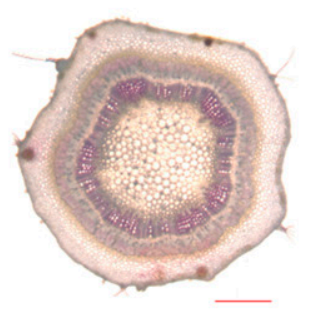

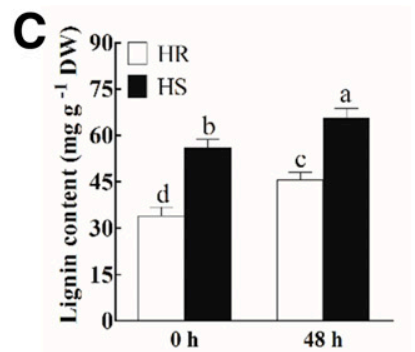

D
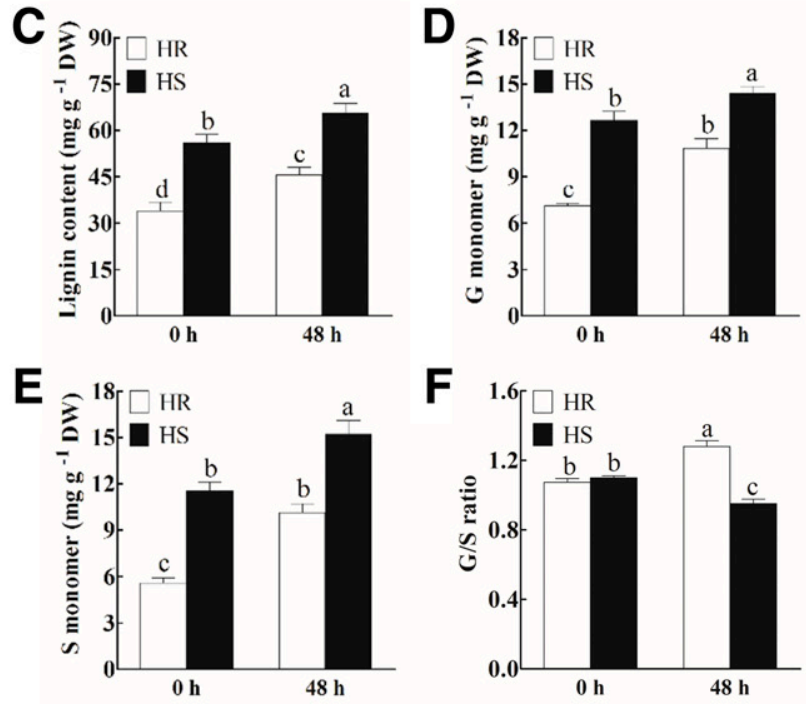

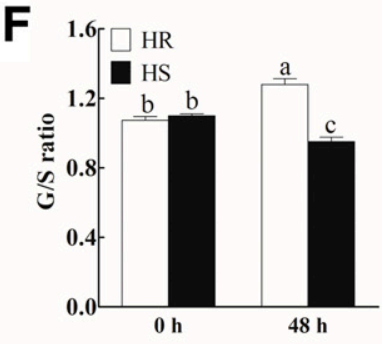

Fig. 3. Expression profiles of the lignin biosynthesis-related genes, comparison of lignin content, and lignin monomer in resistant Shidalukang 1 (HR) and susceptible Junmian 1 (HS) before and after Verticillium dahliae infection. A, Transcript profiling of the 34 differentially expressed genes (DEGs) involved in the lignin biosynthesis pathways in $\mathrm{HR}$ and $\mathrm{HS}$. PAL = phenylalanine ammonia-lyase, $\mathrm{C} 4 \mathrm{H}=$ cinnamate 4-hydroxylase, 4CL $=4$-coumarate: CoA ligase, $\mathrm{C} 3 \mathrm{H}=$ p-coumarate 3 hydroxylase, $\mathrm{HCT}=$ hydroxycinnamoly transferase, $\mathrm{CSE}=$ caffeoyl shikimate esterase, $\mathrm{F} 5 \mathrm{H}=$ ferulate 5 -hydroxylase, $\mathrm{CCR}=$ cinnamoyl CoA reductase, $\mathrm{CAD}=$ cinnamyl alcohol dehydrogenase, $\mathrm{COMT}=$ caffeic acid 3-O-methyltransferase, and CCoAOMT $=$ caffeoyl-CoA O-methyltransferase. B, Histochemical analysis (stained with phloroglucinol-HCl) of lignin in the stem cross-sections of $\mathrm{HR}$ and $\mathrm{HS}$ plants at 0 and $48 \mathrm{~h}$ postinoculation (hpi). Scale bars $=300 \mu \mathrm{m}$. The content of $\mathbf{C}$, total lignin; $\mathbf{D}, \mathrm{G}$ monomer; and $\mathbf{E}$, S monomer in the stems of HR and HS at 0 and 48 hpi. F, The G/S ratio in the stems of HR and HS at 0 and $48 \mathrm{hpi}$. Data are the mean \pm standard error of three independent biological replicates, and different letters indicate significant differences $(P<0.05)$ between groups determined using the Duncan's multiple range test. 
To investigate the role of Gh4CL3O in V. dahliae resistance, we first determined the expression pattern of Gh4CL3O in different tissues of HR and HS. Gh4CL3O had a higher expression level in $V$. dahliae-susceptible varieties than in $V$. dahliae-resistant varieties in all three tissues analyzed. Among the three tissues, the expression level of Gh4CL30 was higher in stems than in roots and leaves (Fig. 5A). Time course expression analysis in both roots and stems revealed that, in response to $V$. dahliae infection, the expression level of Gh4CL30 was significantly upregulated only in roots at 24 hpi and in both roots and stems at 48 and $72 \mathrm{hpi}$ in the two cultivars (Fig. 5B and C). These results suggested that Gh4CL3O might be a negative regulator of $V$. dahliae resistance.

\section{Virus-induced gene silencing mediated silencing of $G h 4 C L 30$ enhanced cotton resistance to $V$. dahliae.}

If $G h 4 C L 30$ acts as a negative regulator of $V$. dahliae resistance, we expected to see enhanced $V$. dahliae resistance in HS when Gh4CL30 is silenced. We used virus-induced gene silencing (VIGS) to silence Gh4CL30 and successfully generated VIGS plants, as demonstrated by the yellowing phenotype observed on the newly developing true leaves in the control TRV:GhCHLI plants (Supplementary Fig. S5). RT-qPCR analysis of Gh4CL30 in TRV:O0 and TRV:Gh4CL3O plants 2 weeks after infiltration confirmed significant reduction of the transcript level of Gh4CL30 in TRV:Gh4CL30 plants (Fig. 6A). Pathogen infection experiments indicated that the $T R V$ : Gh4CL30 plants had a significantly increased level of $V$. dahliae resistance (Fig. 6B), as demonstrated by less severe disease symptoms and diseased plants, and lower disease index was observed in the TRV:Gh4CL30 plants (Fig. 6C and D), as well as fewer fungal colonies recovered from stems of the TRV:Gh4CL30 plants and significantly lower accumulation of fungal biomass in the TRV:Gh4CL30 plants (Fig. 6E and F).

It is noteworthy that stems of the mock $T R V: 00$ plants did not show red-brown color in the xylem, whereas the TRV:Gh4CL30 stems did. Upon $V$. dahliae infection, both TRV:00 and TRV:

Gh4CL30 plants exhibited a red-brown phenotype in the xylem of stems, with a stronger color observed in the TRV:Gh4CL30 plants (Supplementary Fig. S6), and the intensity of the red-brown

Table 1. Candidate hub genes associated with response to Verticillium dahliae inoculation in Shidalukang 1 and Junmian 1

\begin{tabular}{|c|c|c|}
\hline Gene name & Description & KME value ${ }^{a}$ \\
\hline \multicolumn{3}{|l|}{ Purple module } \\
\hline$G h \_D 10 G 0473$ & 4-Coumarate-CoA ligase 1 & 0.92 \\
\hline$G h \_A 13 G 2072$ & Cytochrome P450 98A2 & 0.90 \\
\hline \multicolumn{3}{|l|}{ Brown module } \\
\hline Gh_A01G1981 & Ammonium transporter 2 & 0.94 \\
\hline$G h_{-} A 08 G 1891$ & Glutamate dehydrogenase 3 & 0.90 \\
\hline \multicolumn{3}{|l|}{ Yellow module } \\
\hline$G h \_A 12 G 1327$ & ATP-citrate synthase $\alpha$ chain protein 1 & 0.93 \\
\hline \multicolumn{3}{|l|}{ Blue module } \\
\hline$G h \_D 11 G 1150$ & $\begin{array}{l}\text { DNA replication licensing factor } \\
\text { MCM7 }\end{array}$ & 0.95 \\
\hline$G h \_A 11 G 0031$ & Thaumatin-like protein & 0.95 \\
\hline$G h \_A 11 G 3071$ & $\begin{array}{l}\text { DNA replication licensing factor } \\
\text { MCM7 }\end{array}$ & 0.93 \\
\hline$G h \_D 08 G 0168$ & Origin of replication complex subunit $1 \mathrm{~B}$ & 0.93 \\
\hline Gh_A13G0286 & $\begin{array}{l}\text { DNA replication licensing factor } \\
\text { MCM4 }\end{array}$ & 0.93 \\
\hline Gh_D03G0910 & $\begin{array}{l}\text { Replication protein A } 70-k D a \text { DNA- } \\
\text { binding subunit B }\end{array}$ & 0.92 \\
\hline$G h \_D 05 G 3787$ & PsbP-like protein 1 , chloroplastic & 0.92 \\
\hline$G h \_A 02 G 1196$ & Pleiotropic drug resistance protein 1 & 0.92 \\
\hline$G h \_A 03 G 0625$ & $\begin{array}{l}\text { Replication protein A } 70-\mathrm{kDa} \text { DNA- } \\
\text { binding subunit B }\end{array}$ & 0.91 \\
\hline Gh_A07G1821 & $\begin{array}{l}\text { Cell division control protein } 45 \\
\text { homolog }\end{array}$ & 0.91 \\
\hline \multicolumn{3}{|l|}{ Green module } \\
\hline$G h \_D 06 G 0003$ & Hexokinase-2 & 0.97 \\
\hline$G h \_D 01 G 0800$ & Phosphoglycerate kinase & 0.95 \\
\hline Gh_A05G1529 & $\begin{array}{l}\text { Glyceraldehyde-3-phosphate } \\
\text { dehydrogenase B }\end{array}$ & 0.95 \\
\hline Gh_A10G0618 & $\begin{array}{l}\text { Glyceraldehyde-3-phosphate } \\
\text { dehydrogenase A }\end{array}$ & 0.94 \\
\hline$G h \_A 05 G 1564$ & Plastocyanin A & 0.94 \\
\hline$G h \_A 06 G 0047$ & Hexokinase-2 & 0.93 \\
\hline
\end{tabular}

A

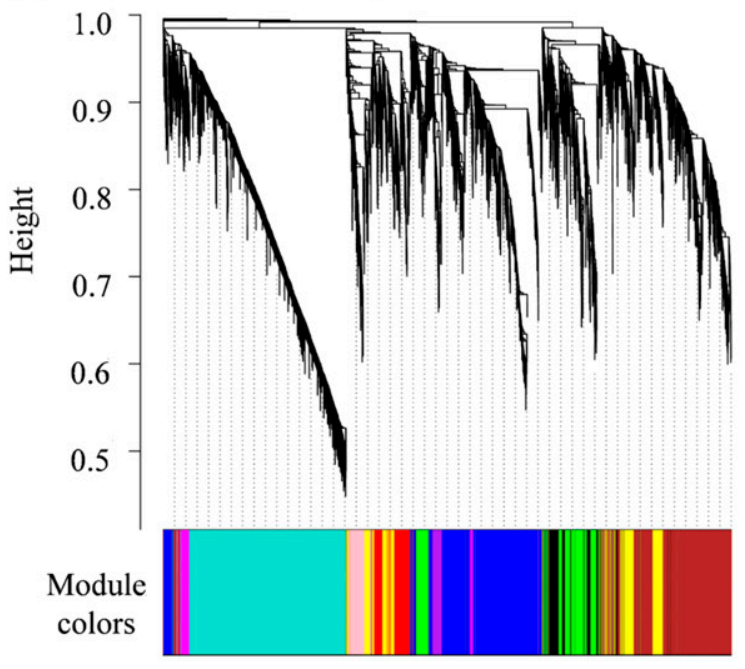

B

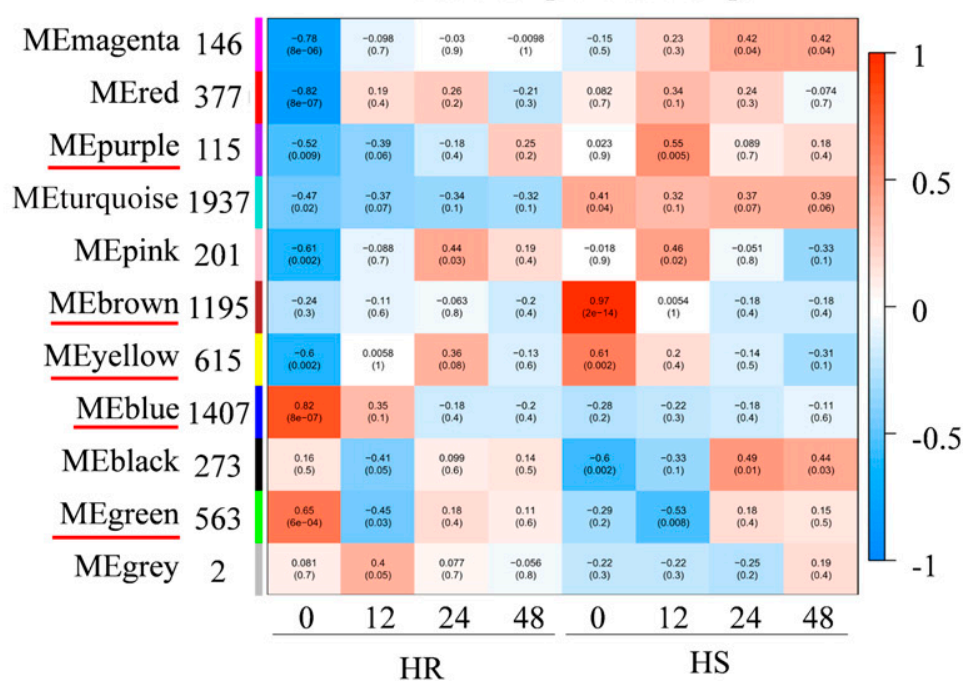

Fig. 4. Weighted gene coexpression network analysis (WGCNA) based on the differentially expressed genes (DEGs) identified between resistant Shidalukang 1 (HR) and susceptible Junmian 1 (HS) at $0 \mathrm{~h}$ postinoculation (hpi) and their expression profiles at 12, 24, and 48 hpi. A, Hierarchical cluster tree showing coexpression modules identified by WGCNA. Each leaf in the tree represents one gene. Each major tree branch corresponds to a distinct module. In total, 6,829 DEGs were grouped into 10 coexpression modules labeled with different colors. The DEGs that did not show a coexpression relationship were assigned into the gray module. B, Relationships between the coexpression modules and samples. Each row corresponds to a module, labeled with the same color as in A. The corresponding correlation and $P$ value were annotated in each cell. Modules that had a high degree of correlation in at least one time point in either of the two varieties are indicated by a red underline. 
color decreased upward in the xylem of stems (Supplementary Fig. S7).

\section{Silencing of $G h 4 C L 30$ had variable effect} on the accumulation of different lignin monomers.

It is known that $4 C L s$ are key genes involved in the biosynthesis of lignins (Vanholme et al. 2010). We compared the lignin content in the stems of the TRV:00 and TRV:Gh4CL3O plants. Phloroglucinol staining of stems suggested that the $T R V$ : Gh4CL30 plants accumulated less lignin than the TRV:00 plants regardless of whether or not the plants were inoculated with V. dahliae (Fig. 7A). Consistently, significantly lower amount of total lignin was detected in the TRV:Gh4CL30 plants than in the TRV:00 plants in both mock and $V$. dahliae inoculation treatments (Fig. 7B). In agreement with these observations, the expression levels of four lignin biosynthesis-related genes, including GhCOMT1, GhCCR1, GhCCR2, and GhCAD1, were significantly downregulated in the TRV:Gh4CL3O plants compared with the TRV:OO plants in both mock and $V$. dahliae inoculation treatments. The expression levels of two other lignin biosynthesis-related genes, GhC4H1 and GhCCoAOMT1, were reduced only in the stems from $V$. dahliae-infected plants but not in the stems from uninfected plants (Fig. 7C to $\mathrm{H}$ ). Given the importance of different lignin monomers in plant defense against $V$. dahliae infection (Xu et al. 2011), we further measured the content of the $\mathrm{G}, \mathrm{S}$, and $\mathrm{H}$ lignin monomers. In both
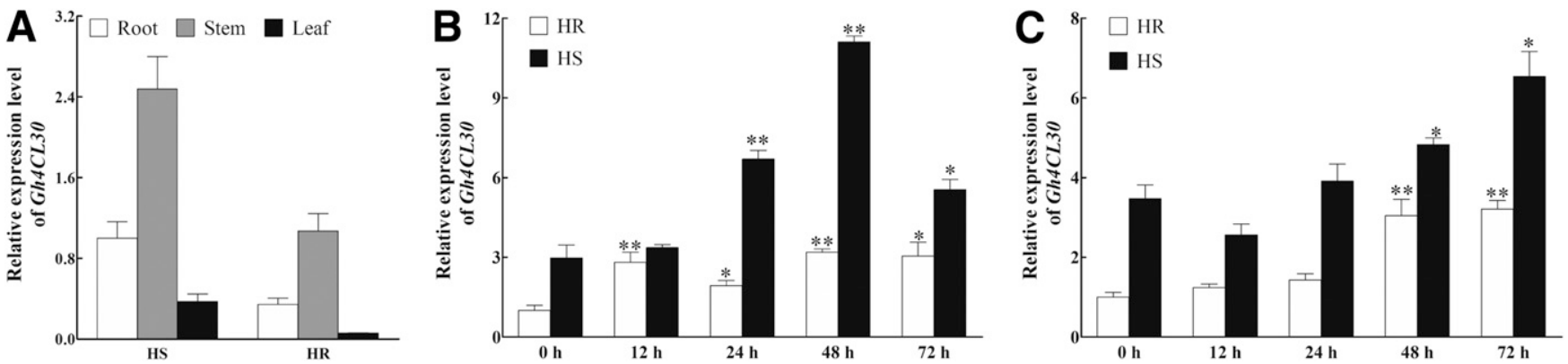

Fig. 5. Expression pattern of Gh4CL30 in different cotton tissues and in response to Verticillium dahliae inoculation. A, Expression profiling of Gh4CL30 in roots, stems, and leaves in resistant Shidalukang 1 (HR) and susceptible Junmian 1 (HS). Roots, stems, and leaves were sampled from two-leaf-stage seedlings grown in a greenhouse. B, Expression patterns of Gh4CL30 in the roots of HS and HR at 0, 12, 24, 48, and $72 \mathrm{~h}$ postinoculation (hpi). GhUBQ7 was used as the control. C, Expression patterns of Gh4CL30 in the stems of HS and HR at 0, 12, 24, 48, and 72 hpi. GhUBQ7 was used as the control. Data are the mean \pm standard error of three independent biological replicates, and asterisks indicate significant differences between groups determined using the Student's $t$ test (* and $* *$ indicate $P<0.05$ and 0.01 , respectively).

A

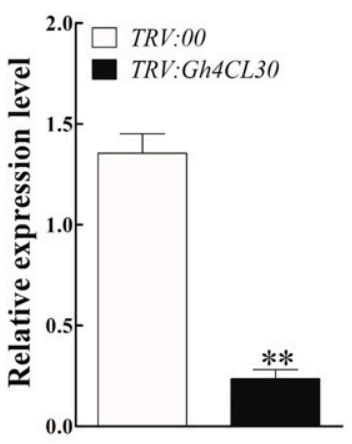

B

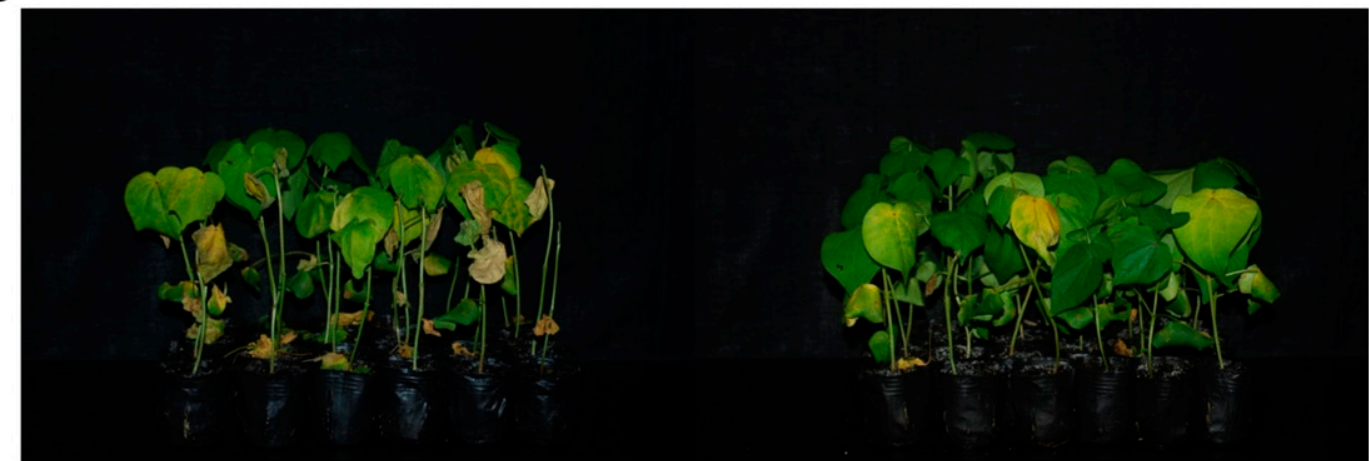

C

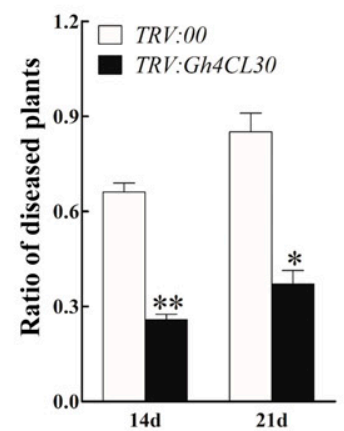

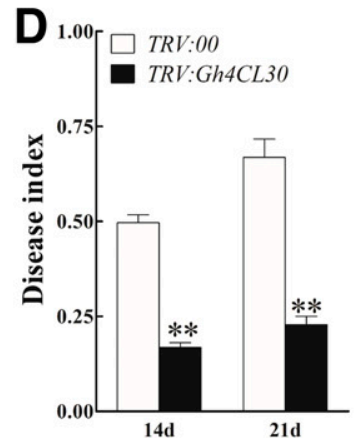
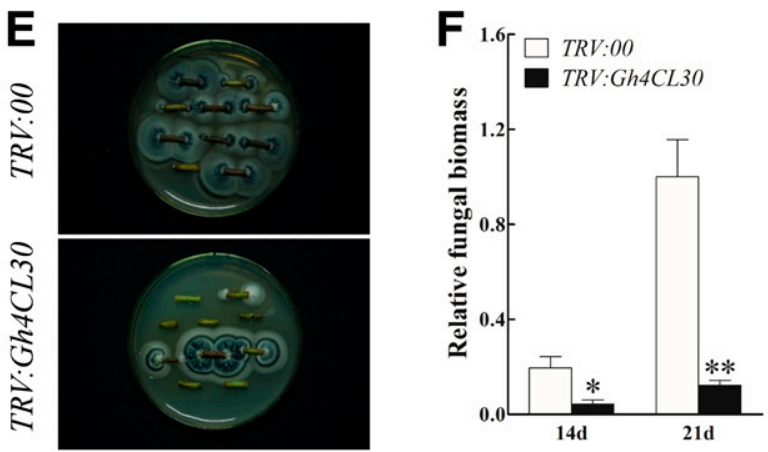

Fig. 6. Silencing of Gh4CL30 in Junmian 1 (HS) improved resistance to Verticillium dahliae. A, Reverse-transcription quantitative PCR (RT-qPCR) analysis of Gh4CL30 expression in the TRV:00 and TRV:Gh4CL30 plants. Total RNA was isolated from stems at 10 days after infiltration with virus-induced gene silencing vector. GhUBQ7 was used as the control. B, Phenotypes of the TRV:00 and TRV:Gh4CL30 plants at 14 days postinfection (dpi). C and D, Ratio of diseased plants and disease index for the TRV:00 and TRV:Gh4CL30 plants at 14 and 21 dpi. E, Fungal isolation in the stem sections from TRV:00 and TRV: Gh4CL3O plants at $14 \mathrm{dpi}$. Stems were plated on potato dextrose agar medium. Photos were taken after 7 days of culture at $25^{\circ} \mathrm{C}$. F, qPCR analysis of the relative fungal biomass in stems of the TRV:00 and TRV:Gh4CL30 plants at 14 and 21 dpi. Data are the mean \pm standard error of three independent biological replicates, and asterisks indicate significant differences between groups determined using the Student's $t$ test (* and $* *$ indicate $P<0.05$ and 0.01 , respectively). 
mock and $V$. dahliae infection treatments, the content and the proportion of the $\mathrm{G}$ unit were higher in the TRV:Gh4CL3O plants than in the TRV:00 plants; by contrast, the content and the proportion of the $\mathrm{S}$ unit were lower in the TRV:Gh4CL3O plants than in the TRV:00 plants. The content and the proportion of the less abundant $\mathrm{H}$ unit remained stable. As a result, the $\mathrm{G} / \mathrm{S}$ ratio was higher in the TRV:Gh4CL30 plants than in the TRV:00 plants, particularly in the $V$. dahliae treatment (Table 2). The
A

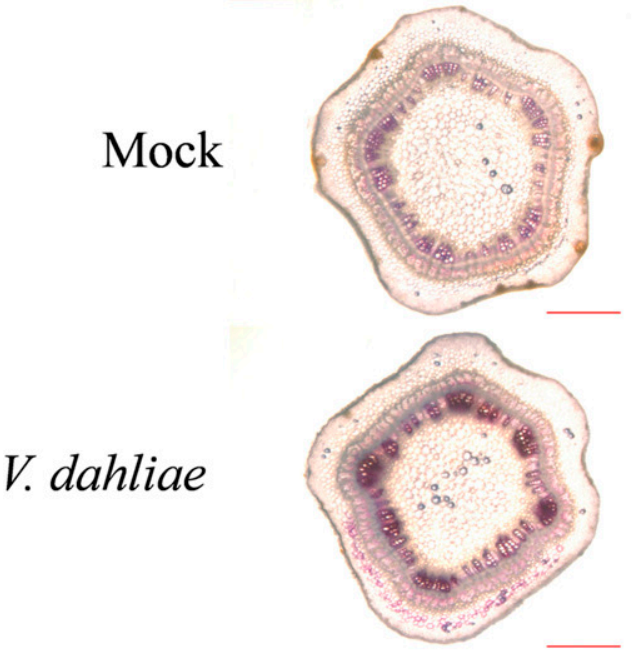

TRV:Gh4CL30

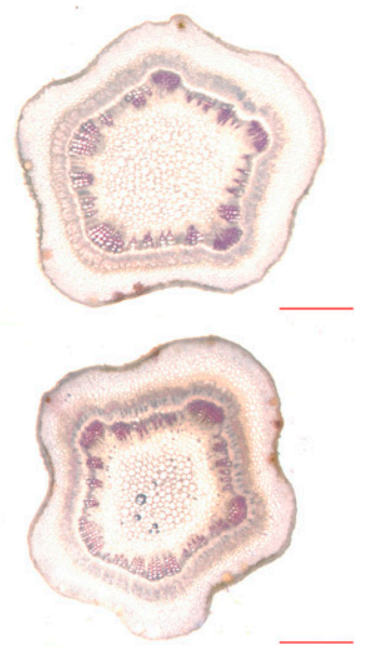

B

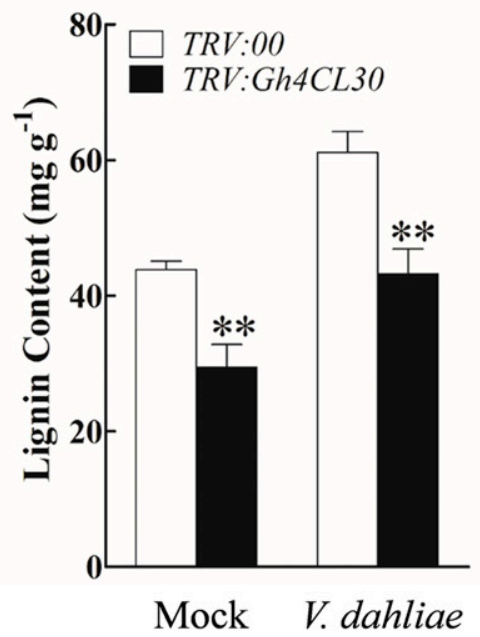

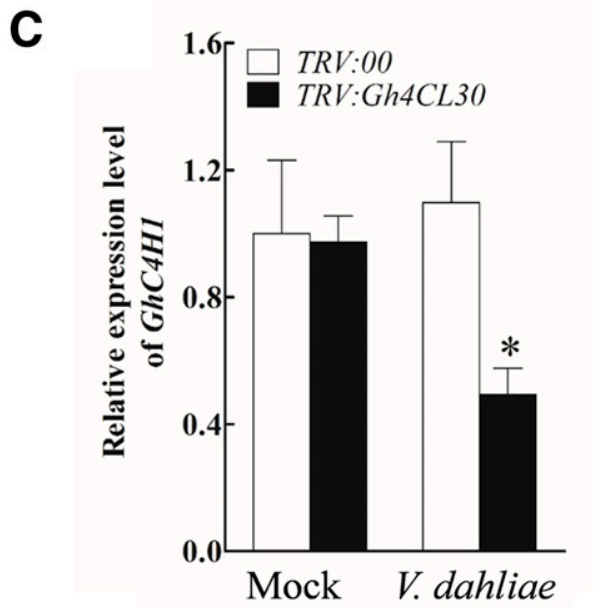
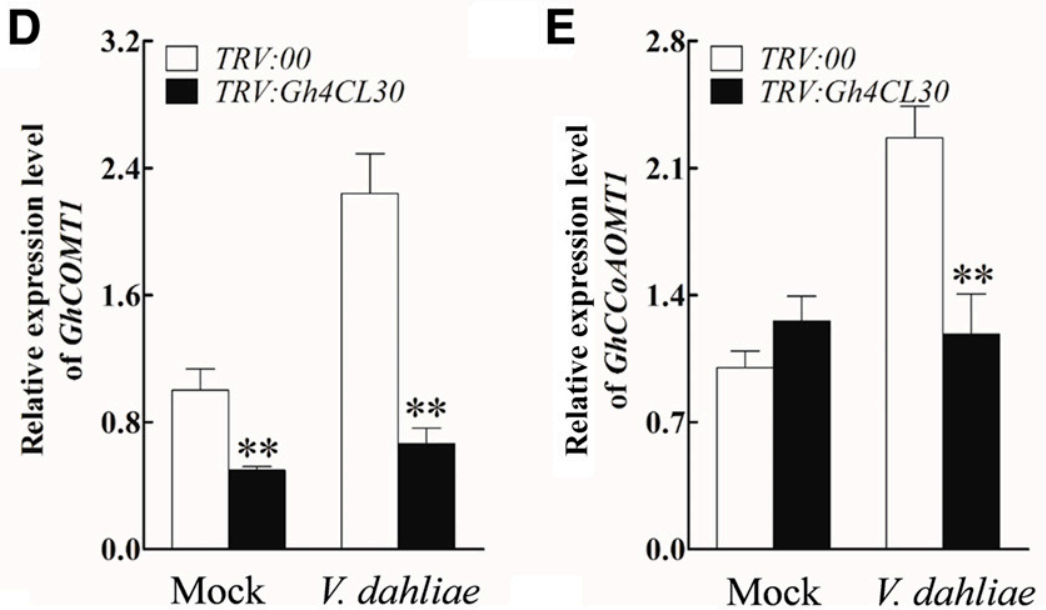

$\mathbf{F}$
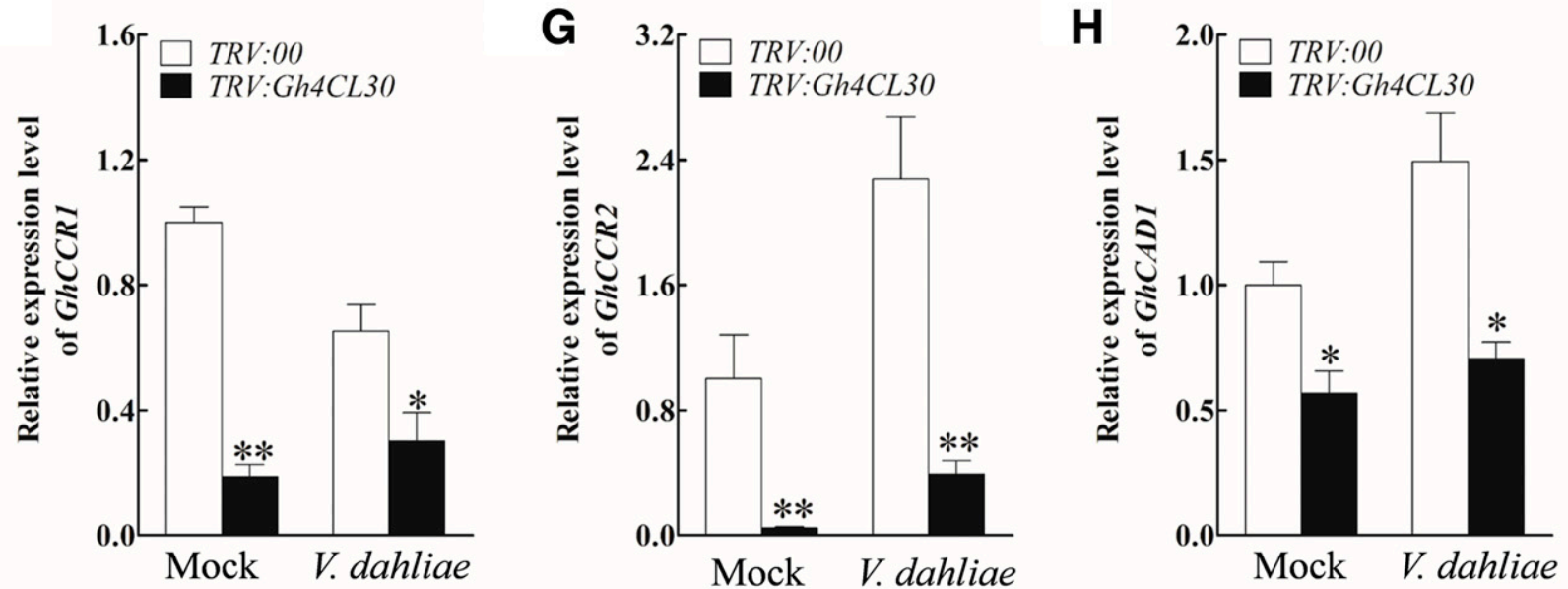

Fig. 7. Comparison of lignin content and expression of lignin biosynthesis-related genes in TRV:00 and TRV:Gh4CL30 plants at $48 \mathrm{~h}$ postinoculation (hpi) (mock versus Verticillium dahliae infection). A, Histochemical analysis of lignin in the stem cross-sections from TRV:00 and TRV:Gh4CL30 plants. The handcut stem was stained with phloroglucinol- $\mathrm{HCl}$ to detect lignin. Scale bars $=300 \mu \mathrm{m}$. B, Comparison of the total lignin content in the stems of TRV:00 and TRV: Gh4CL30 plants after 48-h treatment of $V$. dahliae or sterile distilled water. C to $\mathbf{H}$, Reverse-transcription quantitative PCR (RT-qPCR) analysis of lignin biosynthesis-related genes in TRV:00 and TRV:Gh4CL30 plants after 48-h treatment of V. dahliae or sterile distilled water. Data are the mean \pm standard error of three independent biological replicates, and asterisks indicate significant differences between groups determined using the Student's $t$ test $(*$ and $* *$ indicate $P<0.05$ and 0.01 , respectively). 
decreased total lignin content observed in TRV:Gh4CL30 was mainly due to reduction of the $\mathrm{S}$ unit (Fig. 7B; Table 2). Nevertheless, compared with the mock treatment, $V$. dahliae induced accumulation of lignin in both $T R V: 00$ and $T R V$ : Gh4CL30 plants (Fig. 7B; Table 2). These results suggest that knockdown of the expression level of Gh4CL3O had different effects on the production of the $\mathrm{G}$ and $\mathrm{S}$ lignin monomers but $V$. dahliae infection consistently enhanced the production of all linin monomers.

\section{Silencing of $G h 4 C L 30$ repressed the salicylic acid and jasmonic acid signaling pathways and flavonoid biosynthesis.}

Salicylic acid (SA) and jasmonic acid (JA) are two of the major phytohormones that play a vital role in plant defense against pathogen infection (Shaban et al. 2018). To investigate the effect of silencing Gh4CL30 on the SA and JA biosynthesis or signaling pathways, we compared the SA and JA concentrations in the stems of the TRV:00 and TRV:Gh4CL3O plants. The content of SA was significantly lower in TRV:Gh4CL30 than in TRV:OO in both uninfected and infected treatments (Supplementary Fig. S8A); however, the reduction of JA content in TRV:Gh4CL30 was observed only in the mock treatment (Supplementary Fig. S8B). Compared with the TRV:00 plants, the TRV:Gh4CL30 plants had a variable change in the expression level of the SA signaling or biosynthesis genes in the mock treatment but showed a consistently significant reduction of all four genes upon $V$. dahliae infection (48 hpi) (Supplementary Fig. S8C to F). The two JA biosynthesis or signaling genes (GhAOS1 and GhPR4) were significantly inhibited in the TRV: Gh4CL30 plants compared with the TRV:00 plants in both the mock and $V$. dahliae infection treatments (Supplementary Fig. $\mathrm{S} 8 \mathrm{G}$ and $\mathrm{H}$ ). These results suggest that downregulation of Gh4CL30 tends to repress accumulation of SA and JA that is further affected by $V$. dahliae infection.

4CLs are also involved in the biosynthesis of flavonoids (Lavhale et al. 2018). Previous studies have reported that increased contents of flavonoids inhibited colony growth of $V$. dahliae (Hu et al. 2018). We found that the diameters of $V$. dahliae colonies growing on the medium treated with the methanolic extracts from the TRV:Gh4CL30 plants were significantly smaller than those treated with the extracts from the TRV:00 plants (Fig. 8A and B). However, we observed a significant decrease of flavonoids in the TRV:Gh4CL3O plants compared with the TRV:00 plants in both the mock and $V$. dahliae inoculation treatments (Supplementary Fig. S9A). The expression levels of four flavonoid biosynthesis-related genes, including GhANR1, GhCHII, GhLARl, and GhANS1, were significantly reduced in the $V$. dahliae-infected $T R V$ :
Gh4CL30 plants, although no significant difference was evident between the uninfected TRV:Gh4CL3O and TRV:0O plants (Supplementary Fig. S9B to E). These results suggest that the substances from TRV:Gh4CL30 that inhibited the growth of the $V$. dahliae colony are most likely not flavonoids.

\section{Altered phenolic metabolism in the Gh4CL30-silenced plants was associated with resistance to $V$. dahliae.}

Reports have suggested that the phenolic precursors of lignin biosynthesis, including ferulic acid and caffeic acid, have antifungal effects (Lanoue et al. 2010; Lavhale et al. 2018). Thus, we quantified the content of four phenolics in the lignin biosynthesis pathway, including cinnamic acid, coumalic acid, caffeic acid, and ferulic acid, in the stems of the TRV:00 and TRV:Gh4CL3O plants. Under the mock treatment, the contents of ferulic acid and caffeic acid were 9.0- and 6.1-fold higher, respectively, in TRV:Gh4CL30 than in TRV:00 (Fig. 8C and D). At 48 hpi, TRV:Gh4CL30 showed an even higher increase of the two phenolics, at 10.4- and 32.3fold for ferulic acid and caffeic acid, respectively (Fig. 8C and D). No significant change was observed for coumalic acid and cinnamic acid (Fig. 8E and F). These results suggest that accumulation of ferulic acid and caffeic acid might be associated with the enhanced $V$. dahliae tolerance observed in TRV:Gh4CL3O.

We investigated the effect of ferulic acid and caffeic acid on the growth of $V$. dahliae (strain V991) using four different concentrations $\left(10 \mu \mathrm{g} \mathrm{ml}^{-1}, 100 \mu \mathrm{g} \mathrm{ml}^{-1}, 1 \mathrm{mg} \mathrm{ml}^{-1}\right.$, and $10 \mathrm{mg}$ $\mathrm{ml}^{-1}$ ) of these two phenolics. Three of the four concentrations (except $10 \mu \mathrm{g} \mathrm{ml}^{-1}$ ) were found to significantly inhibit the growth of $V$. dahliae colony, and the higher the concentration, the stronger the inhibition (Fig. 8G and H; Supplementary Fig. S10). Furthermore, a mixture of ferulic acid and caffeic acid seemed to have a stronger inhibition compared with either single phenolic (Fig. 8G and H; Supplementary Fig. S10). These findings indicated that the higher levels of ferulic acid and caffeic acid contribute to the increased resistance level of the TRV:Gh4CL30 plants against $V$. dahliae infection.

\section{DISCUSSION}

In this study, we used comparative transcriptomics to investigate the molecular mechanism underlying the basal and pathogen-induced responses in the interaction between cotton and $V$. dahliae. We identified Gh4CL3O to be one of the hub genes that were differentially expressed between cultivars resistant or susceptible to V. dahliae. Gh4CL30 regulates lignification and phenolic content and negatively contributes to $V$. dahliae resistance. We also found that a higher ratio of the $\mathrm{G} / \mathrm{S}$ lignin monomer is more important than the total lignin content in cotton resistance to $V$. dahliae. The present study

Table 2. Comparison of individual lignin monomers in the Gh4CL30-silenced and TRV:00 plants in Junmian $1^{\mathrm{x}}$

\begin{tabular}{|c|c|c|c|c|c|c|c|c|}
\hline \multirow[b]{2}{*}{ Sample } & \multicolumn{3}{|c|}{$\begin{array}{c}\text { Monomer } \\
\text { composition (mg } \mathrm{g}^{-1} \text { dry weight) }\end{array}$} & \multirow[b]{2}{*}{ Lignin $^{y}$} & \multicolumn{3}{|c|}{$\begin{array}{l}\text { Ratio of monomer } \\
\text { composition }\end{array}$} & \multirow[b]{2}{*}{$\mathbf{G} / \mathbf{S}^{\mathbf{z}}$} \\
\hline & $\mathbf{G}$ & $\mathbf{S}$ & $\mathbf{H}$ & & G & $\mathbf{S}$ & $\mathbf{H}$ & \\
\hline \multicolumn{9}{|l|}{ Mock } \\
\hline$T R V: 00$ & $11.50 \pm 0.25 c$ & $10.39 \pm 0.15 b$ & $0.54 \pm 0.05 \mathrm{~b}$ & $22.43 \pm 0.65 b$ & 0.51 & 0.46 & 0.02 & 1.11 \\
\hline TRV:Gh4CL30 & $12.43 \pm 0.28 b$ & $8.49 \pm 0.15 c$ & $0.62 \pm 0.02 \mathrm{a}$ & $21.53 \pm 0.41 c$ & 0.58 & 0.39 & 0.03 & 1.46 \\
\hline \multicolumn{9}{|l|}{ Inoculated } \\
\hline$T R V: 00$ & $12.20 \pm 0.14 b$ & $12.55 \pm 0.23 \mathrm{a}$ & $0.52 \pm 0.02 b$ & $25.27 \pm 0.71 \mathrm{a}$ & 0.48 & 0.50 & 0.02 & 0.97 \\
\hline$T R V: G h 4 C L 30$ & $13.71 \pm 0.34 \mathrm{a}$ & $8.95 \pm 0.21 c$ & $0.53 \pm 0.02 b$ & $23.19 \pm 0.44 b$ & 0.59 & 0.39 & 0.02 & 1.53 \\
\hline
\end{tabular}


improves our understanding of the functions of Gh4CL30 and provides new insights about the resistance mechanism employed by cotton against $V$. dahliae.

Both basal and pathogen-induced transcriptomes play an important role in cotton defense against $V$. dahliae.

When doing transcriptome comparison, most researches usually focus on differentially expressed genes between infected and uninfected samples of resistant and susceptible cultivars (Gao et al. 2013; Sun et al. 2013; Xu et al. 2011, 2014; Zhang et al. 2013), and the basal transcriptomic difference between susceptible and resistant cultivars is largely overlooked. In a recent study in olive, the $V$. dahliae tolerance and susceptibility were linked to differences in the basal transcriptomes (Leyva-Pérez et al. 2018). Here, we found 6,831 DEGs in uninfected roots and stems between HR and HS (Fig. 2A; Supplementary Tables S4 and S5), of which 2,866 and 3,965 had expression levels higher in HR and HS, respectively (Fig. 2D; Supplementary Tables S4 and S5). These basal DEGs may contribute to the resistance and susceptibility of HR and
HS. Many genes highly expressed in uninfected HR were involved in response to stimulus and stress (Fig. 2C), including two ABC transporters, two major latex proteins, one wallassociated receptor-like kinase, and several disease resistance proteins. Consistently, recent studies showed that silencing GhABCF5 or GhMLP28 in cotton led to a significantly decreased resistance to $V$. dahliae (Dong et al. 2019; Yang et al. $2015)$. The plants with a mutation in the gene ( $Z m W A K-R L K 1)$ encoding a wall-associated receptor-like kinase were more susceptible to northern corn leaf blight than wild-type plants in both the field and greenhouse conditions (Hurni et al. 2015).

Numerous studies have demonstrated that plants restrict pathogen spread in the vascular structure by changing the lignin monomer composition and lignification ( $\mathrm{Hu}$ et al. 2018; $\mathrm{Xu}$ et al. 2011). We also found a large number of lignin biosynthesis-related genes that were significantly induced in both HR and HS after V. dahliae infection (Supplementary Figs. S3 and S4). Consequently, both HR and HS plants showed a higher level of total lignin, due to accumulation of the $\mathrm{G}$ and $\mathrm{S}$ monomers, after $V$. dahliae infection (Fig. 3B to E). Similar
A
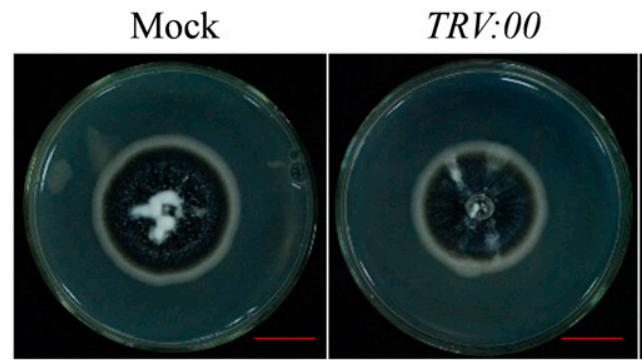

TRV:00

TRV:Gh4CL30

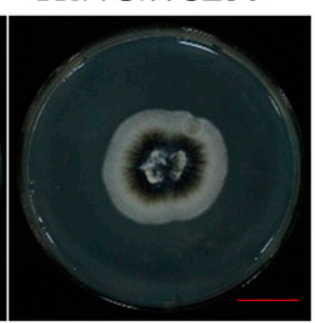

B

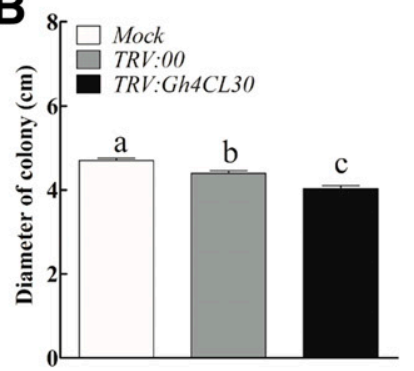

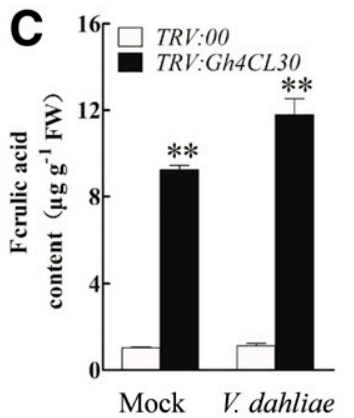

G

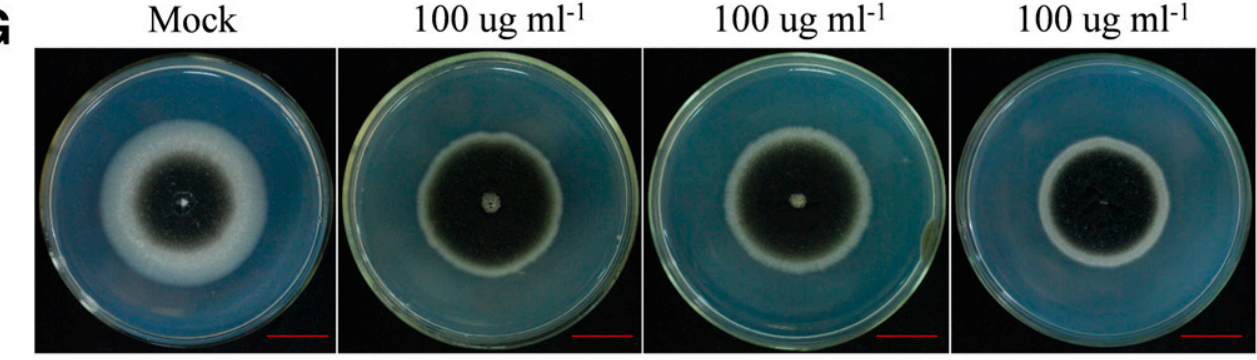

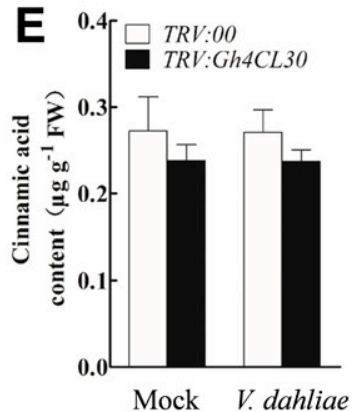
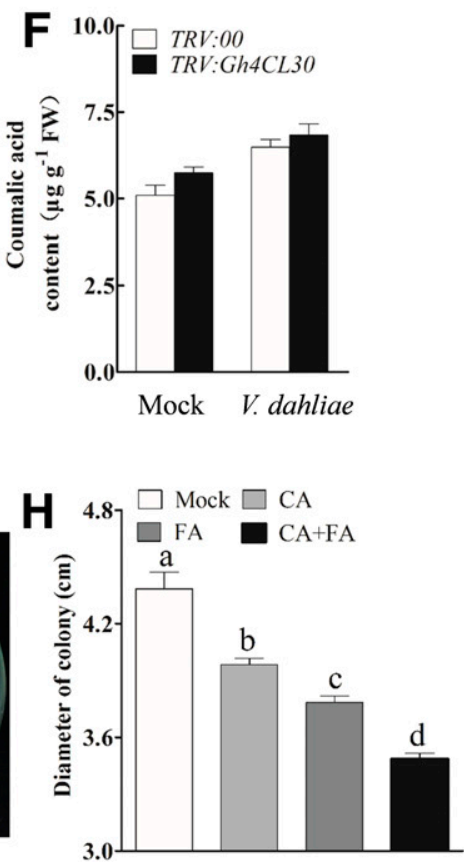

Fig. 8. Silencing Gh4CL30 enhances cotton resistance to Verticillium dahliae correlates with accumulation of caffeic acid and ferulic acid. A and B, The fungus was grown for 10 days on potato dextrose agar (PDA) plates with methanolic extracts (the final concentration was $2 \%$ [vol/vol]) from the stems of $T R V: 00$ and TRV:Gh4CL30 plants and the diameter of the colony was determined. Scale bars $=2 \mathrm{~cm}$. Data are the mean \pm standard error (SE) of three independent biological replicates, and different letters indicate significant differences $(P<0.05)$ between groups determined using the Duncan's multiple range test. Comparison of $\mathbf{C}$, ferulic acid; D, caffeic acid; E, cinnamic acid; and F, coumalic acid in the stem of TRV:00 and TRV:Gh4CL30 plants after 48-h treatment of $V$. dahliae or sterile distilled water. Data are the mean $\pm \mathrm{SE}$ of three independent biological replicates, and asterisks indicate significant differences between groups determined using the Student's $t$ test (* and ** indicate $P<0.05$ and 0.01 , respectively). G and $\mathbf{H}$, Effects of ferulic acid (FA) and caffeic acid (CA) on colony growth $(n=5)$. The photograph was taken after growing the fungus for 12 days on the PDA plates with FA or CA at $100 \mu \mathrm{g} \mathrm{m}^{-1}$. Scale bars $=2 \mathrm{~cm}$. Data are the mean \pm SE of three independent biological replicates, and different letters indicate significant differences $(P<0.05)$ between groups determined using the Duncan's multiple range test. 
results were reported in the $V$. dahliae-tolerant $G$. barbadense accession and V. dahliae-susceptible G. hirsutum accession (Xu et al. 2011). These findings suggest that activation of the lignin biosynthesis pathways is associated with pathogen-induced resistance in both $V$. dahliae-resistant and -susceptible cotton. Previous studies showed that resistant cotton plants accumulated more lignin than susceptible cotton plants after $V$. dahliae inoculation and that the lignin content was positively correlated with $V$. dahliae resistance (Xu et al. 2011; Zhang et al. 2019). However, we found that HR had a significantly lower level of lignin than HS in both mock and $V$. dahliae infection treatments, despite $V$. dahliae-induced accumulation of lignin (Fig. $3 \mathrm{~B}$ to E). Similarly, decreased lignin content but increased disease resistance was also observed among alfalfa, Arabidopsis, and cotton (Gallego-Giraldo et al. 2011, 2020; Hu et al. 2018). Thus, the relationship between the total lignin content and $V$. dahliae resistance is yet to be further investigated using more cotton accessions with different levels of $V$. dahliae resistance.

\section{Silencing Gh4CL30 alters lignin biosynthesis.}

WGCNA is an effective way to identify candidate hub genes and has been successfully used in strawberry (Hollender et al. 2014), soybean (Du et al. 2017), rice (Tan et al. 2017), and cotton (Sun et al. 2019). In this study, we identified several candidate hub genes associated with $V$. dahliae resistance based on WGCNA, including Gh4CL30 that is related to lignin biosynthesis. Several $4 C L s$ genes have been reported to be responsive to pathogen stresses (Ehlting et al. 1999; Oliveira et al. 2015; Sun et al. 2013; Xu et al. 2011). In Arabidopsis, upon Peronospora parasitica infection, the expression of At4CLI and At4CL2 was strongly increased at 12 and 24 hpi (Ehlting et al. 1999). Pv4CL was upregulated in dry bean plants infected by Sclerotinia sclerotiorum (Oliveira et al. 2015). Similarly, $G b 4 C L$ was induced by $V$. dahliae infection in G. barbadense (Sun et al. 2013; Xu et al. 2011). Following V. dahliae inoculation, we observed a consistently significant increase of Gh4CL3O in the roots and stems of the two cotton cultivars used in this study (Fig. 5B and C). Gh4CL30 is related to lignin biosynthesis and a hub gene of the network related to disease response in HR and HS; thus, we further characterized its function in $V$. dahliae resistance. Knockdown of the expression of Gh4CL30 by VIGS led to enhanced resistance to V. dahliae in HS (Fig. 6), suggesting a negative regulatory role of Gh4CL3O in V. dahliae resistance. Similarly, a 4CL-like gene, $O s A A E 3$, has been shown to negatively mediate blast resistance in rice (Liu et al. 2017).

Phylogenetic analysis revealed that Gh4CL30 is more closely related to Arabidopsis At4CL1 and At4CL2 than to At4CL3 and At4CL4 (Feng et al. 2017), suggesting a function of Gh4CL30 similar to that of At4CL1 and At4CL2, which contribute to lignin biosynthesis in the xylem ( $\mathrm{Li}$ et al. 2015). As the key gene in lignin biosynthesis, the function of $4 C L$ in lignin biosynthesis has been extensively investigated in Arabidopsis, rice, and poplar (Ehlting et al. 1999; Gui et al. 2011; Li et al. 2015; Voelker et al. 2010). Similar to what has been found in these plants, downregulation of Gh4CL3O resulted in a decreased level of lignin (Fig. 7A and B), most likely due to a downregulated level of lignin biosynthesis-related genes (Fig. 7C to $\mathrm{H})$; however, it is unexpected that we did not observe decreased disease resistance in the Gh4CL30-silenced plants (Fig. 6). It has been suggested that both the contents of the $G$ and $S$ monomers and the $\mathrm{G} / \mathrm{S}$ ratio could be correlated to disease resistance (Gayoso et al. 2010; Xu et al. 2011). The G/S ratio was increased and decreased in $V$. dahliae-resistant and -susceptible cotton varieties, respectively (Gayoso et al. 2010; Xu et al. 2011). Consistently, we found that the G/S ratio was lower in the $V$. dahliae-infected TRV:OO and HS plants than in the uninfected TRV:00 and HS plants (Fig. 3F; Table 2). However, in the Gh4CL30-silenced and HR plants, an increased G/S ratio was observed after $V$. dahliae infection (Fig. 3F; Table 2), similar to what has been observed in the $V$. dahliae-resistant cotton (Gayoso et al. 2010; Xu et al. 2011). This suggests that the $\mathrm{G} / \mathrm{S}$ ratio rather than the total lignin content determines $V$. dahliae resistance in cotton and explains why plants with a decreased level of total lignin level showed enhanced V. dahliae resistance.

\section{Silencing of Gh4CL30 represses the SA and JA biosynthesis or signaling pathways and flavonoid biosynthesis.}

In Medicago sativa, downregulation of HCT led to decreased lignin content and increased accumulation of SA and JA, and enhanced resistance to Colletotrichum trifolli (Gallego-Giraldo et al. 2011). Methyl jasmonate and SA regulate the expression level of 4CL (Gao et al. 2015; Lee and Douglas 1996), and both SA and JA have been shown to play a major role in the cotton- $V$. dahliae interaction (Shaban et al. 2018). We were interested to know whether Gh4CL30 regulates $V$. dahliae resistance through the SA and JA biosynthesis or signaling pathways. We found that silencing Gh4CL30 reduced SA content in both uninfected and infected plants but reduced JA content only in the uninfected plants (Supplementary Fig. S8A and B), suggesting that $G h 4 C L 30$-silencing-induced reduction of JA biosynthesis could be compensated for by other pathways induced by $V$. dahliae infection, and that the SA and JA biosynthesis pathways may not be the major contributors of the improved $V$. dahliae resistance observed in Gh4CL30-silenced plants.

4CL has also been shown to be important in the biosynthesis of flavonoids (Lavhale et al. 2018). The role of flavonoids on plant-pathogen interactions, by which they can inhibit the growth of pathogens, is well documented (Dixon and Steele 1999; Gill et al. 2018). A recent study in cotton showed that silencing a lignin biosynthesis-related gene, GhLACl, results in decreased total lignin content while increasing accumulated flavonoids and enhanced plant resistance to V. dahliae (Hu et al. 2018). We found that the Gh4CL30-silenced plants produced fewer total flavonoids compared with the TRV:00 plants but the methanolic extracts from the stems of the Gh4CL30-silenced plants could significantly inhibit $V$. dahliae hyphal growth (Fig. 8A and B; Supplementary Fig. S9A and B), suggesting that Gh4CL30 may positively regulate biosynthesis of flavonoids, which is different from what has be reported for the $4 \mathrm{cll}$ mutant in Arabidopsis (Li et al. 2015); however, flavonoids may not be the metabolites contributing to the enhanced $V$. dahliae resistance observed in the Gh4CL30-silenced plants.

\section{Accumulation of phenolics induced by silencing of $G h 4 C L 30$ contributes to increased cotton resistance to $V$. dahliae.}

It is well known that deposition of phenolics acts as an important physical and biochemical barrier contributing to plant defense against vascular pathogens (Eynck et al. 2009; Xu et al. 2011). Downregulating the expression of $4 C L$ could lead to the accumulation of soluble phenolic compounds that are toxic to some pathogens (Miedes et al. 2014). Similar to the results reported in studies on the effects of downregulation of $4 C L 1$ in several other species (Voelker et al. 2010; Zhou et al. 2015), we observed red-brown xylem and increased content of phenolics in the Gh4CL30-silenced plants (Supplementary Figs. S6 and S7; Fig. 8C and D). Cinnamic acid, coumalic acid, caffeic acid, and ferulic acid are the four major phenolics catalyzed by 4CL for monolignin biosynthesis in higher plants, and they have antifungal and antibacterial functions (Boutigny et al. 2009; 
Lanoue et al. 2010; Lavhale et al. 2018). Our previous study found that Gh4CL30 exhibited activity toward cinnamic acid, coumalic acid, caffeic acid, and ferulic acid, with the preferred substrate being ferulic acid (Feng et al. 2017). The abundance of caffeic acid and ferulic acid was significantly increased while that of cinnamic acid and coumalic acid was unchanged in stems of the Gh4CL30-silenced plants compared with that of $T R V: 00$ (Fig. $8 \mathrm{C}$ and D), suggesting that caffeic acid and ferulic acid might be the prominent substances of Gh4CL30. We further demonstrated the role of caffeic acid and ferulic acid in inhibition of $V$. dahliae hyphal growth (Fig. 8G and H), consistent with the results observed in other studies (Boutigny et al. 2009; Dimkić et al. 2016; Dixon 2001). These results indicated that accumulation of caffeic acid and ferulic acid caused by silencing of Gh4CL3O is responsible for the increased $V$. dahliae resistance of the Gh4CL30-silenced plants.

\section{MATERIALS AND METHODS}

\section{Plant materials and culture conditions.}

$V$. dahliae-resistant G. hirsutum 'Shidalukang 1' (HR) and $V$. dahliae-susceptible G. hirsutum 'Junmian 1' (HS) were provided by the Cotton Research Institute of Shihezi University and used for RNA-seq and VIGS experiments (Liu et al. 2002). Cotton seed were sterilized with $0.1 \%$ (wt/vol) $\mathrm{HgCl}_{2}$ for $10 \mathrm{~min}$, then rinsed three times with sterilized distilled water. Cotton seedlings used for all the experiments were grown in a greenhouse under conditions of 24 and $22^{\circ} \mathrm{C}$ day and night temperatures, respectively; a photoperiod of $16 \mathrm{~h}$ of light and $8 \mathrm{~h}$ of darkness; $50 \%$ relative humidity; and average daylight intensity of $240 \mu \mathrm{mol} \mathrm{m} \mathrm{m}^{-2} \mathrm{~s}^{-1}$.

\section{Pathogenicity assays.}

V991, a highly aggressive defoliating $V$. dahliae strain, was kindly provided by Associate Professor Yanfei Sun (Shihezi University). The fungi were subcultured on potato dextrose agar (PDA) medium at $25^{\circ} \mathrm{C}$ in darkness for 7 days. Colonies were then inoculated into Czapek medium for 7 days at $25^{\circ} \mathrm{C}$ with shaking $(150 \mathrm{rpm})$. Two-leaf-stage seedlings were inoculated with $10 \mathrm{ml}$ of

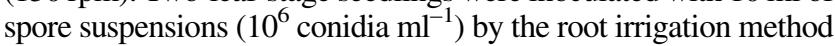
(Xiong et al. 2019). Seedlings inoculated with sterile water were used as mock controls. After inoculation, plants were transferred to a greenhouse. Samples were harvested at 0,12, 24, and $48 \mathrm{hpi}$ from five cotton plants. For each sample, a mixture of $200 \mathrm{mg}$ of roots and $200 \mathrm{mg}$ of stems was used in RNA extraction and RNAseq. Three biological replicates were sampled for each combination of genotype and time point.

\section{RNA extraction, library construction, and sequencing.}

Twenty-four samples (four time points by two cultivars by three biological replicates) from HR and HS were used for RNA-seq. Total RNA was isolated from each sample using a plant RNA Purification Kit (Tiangen, Beijing) according to the manufacturer's instructions. RNA integrity and purity were assessed using the RNA Nano 6000 Assay Kit with the Bioanalyzer 2100 system (Agilent Technologies, Santa Clara, CA, U.S.A.) and a NanoPhotometer spectrophotometer (Implen, Westlake Village, CA, U.S.A.), respectively. A total amount of $3 \mu \mathrm{g}$ of RNA per sample was used in preparation of RNA-seq libraries that were generated using the NEBNext Ultra RNA Library Prep Kit for Illumina (New England Biolabs, Ipswich, MA, U.S.A.) following the manufacturer's recommendations. RNA-seq (150-bp paired-end reads) was performed by Novogene (Novogene, Tianjin, China) on an Illumina Hiseq 4000 platform. The raw RNA-seq data are available in the NCBI under accession number PRJNA593765.

\section{Identification and functional annotation of DEGs.}

Clean reads were generated using in-house Perl scripts by removing reads containing adapter or ploy-N as well as with a phred quality score $<20$ at each nucleotide from the raw data. Paired-end clean reads were aligned to the G. hirsutum reference genome (Zhang et al. 2015) using TopHat v2.0.12.

Gene expression levels were determined by fragments per kilobase of transcript sequence per million base pairs sequenced (FPKM) (Trapnell et al. 2010). Differentially expressed genes were identified by the DESeq R package (1.18.0) (Wang et al. 2010 ) using the criterion of an adjusted $P$ value $<0.05$ (Anders and Huber 2010). DEGs were subjected to GO enrichment analysis by Blast $2 \mathrm{GO}$ with a false discovery rate $<0.05$ (Young et al. 2010). Additionally, KEGG pathways of DEGs were tested for statistical enrichment using the KOBAS 2.0 software with a $P$ value $<0.05$ (Kanehisa et al. 2008). The Venn diagram and heat map were generated by the Novemagic server.

\section{WGCNA analysis.}

Coexpression networks were constructed as described previously using the WGCNA (v1.29) package in R (Langfelder and Horvath 2008). The analysis was based on the FPKM of the 6,831 DEGs. The modules were generated with the default settings, except that the power was set to 14, TOMType was signed, minModuleSize was 30, and merge CutHeight was 0.25 . Each module was represented by a different color.

\section{RT-qPCR analysis.}

cDNA was synthesized from $3 \mu \mathrm{g}$ of total RNA using the MMLV reverse transcription (TaKaRa, Dalian, China). RT-qPCR analyses were conducted using the SYBR Green (Roche, Rotkreuz, Switzerland) on Light Cycler 480II (Roche). All primers used in RT-qPCR were designed using Primer3 Plus and are listed in Supplementary Table S1. The cotton $U B Q 7$ gene (DQ116441.1) was used as an internal control to normalize the variance among samples. PCR conditions consisted of an initial denaturation step at $95^{\circ} \mathrm{C}$ for $5 \mathrm{~min}$ and 40 cycles of denaturation at $95^{\circ} \mathrm{C}$ for $15 \mathrm{~s}$, annealing at $60^{\circ} \mathrm{C}$ for $15 \mathrm{~s}$, and extension at $72^{\circ} \mathrm{C}$ for $20 \mathrm{~s}$. The relative expression level from three biologically independent experiments was evaluated using the $2^{-\Delta \Lambda \mathrm{CT}}$ method (Livak and Schmittgen 2001).

\section{VIGS experiments.}

Tobacco rattle virus (TRV) vectors were used for VIGS experiments. GhCHLI (encoding magnesium chelatase subunit I) was used as a positive control to check the silencing efficiency. The TRV empty vector (TRV:00) was employed as the negative control. Approximately 400-bp fragments were amplified from Gh4CL30 and GhCHLI using the primers listed in Supplementary Table S1 and were then used to construct TRV: Gh4CL3O and TRV:GhCHLI vectors, respectively. The TRVmediated gene silencing in cotton was performed as described previously (Gao et al. 2013). RNA was extracted from cotton stems to measure target gene expression when TRV:GhCHLI cotton plants exhibited yellowing, approximately 10 days after TRV infection.

\section{Characterization of $V$. dahliae resistance in VIGS cotton.}

Two-leaf-stage TRV:Gh4CL3O and TRV:00 cotton plants were inoculated with $10-\mathrm{ml} V$. dahliae spore suspensions $\left(10^{6}\right.$ conidia $\mathrm{ml}^{-1}$ ) by the root irrigation method (Xiong et al. 2019). At least 30 cotton seedlings of similar size were used in each treatment. The ratio of diseased plants and disease index were investigated at 14 and $21 \mathrm{dpi}$. To compare the vascular phenotype, the stems above cotyledons of the infected seedlings were cut at 14 dpi and observed by using a stereo microscope (SteREO Discovery.V20; Zeiss). Fungal biomass quantification 
and fungal recovery assays were performed as previously described (Atallah et al. 2007). Briefly, total DNA was isolated from stems of the infected cotton plants at $14 \mathrm{dpi}$. Primers for the cotton $U B Q 7$ gene were used to quantify cotton DNA, and $V$. dahliae-specific primers ITS1-F and ST-Ve1-R were used to quantify fungal DNA (Supplementary Table S1) (Ellendorff et al. 2009). For stem culture, the stem section above the cotyledons was collected from 10 infected plants at $14 \mathrm{dpi}$. The sections were surface sterilized in 3\% hypochlorite for $3 \mathrm{~min}$ and rinsed three times with sterile water, then cut into $1-\mathrm{cm}$ slices and plated on PDA medium at $25^{\circ} \mathrm{C}$ in darkness.

\section{Lignin histochemical staining.}

Visualization of histochemical staining of lignin was done using the Wiesner staining method (Xu et al. 2011). At 48 hpi, stems at the same position were collected from mock- and $V$. dahliae-inoculated HR, HS, TRV:00, and TRV:Gh4CL30 plants, hand cut into slices, and dipped in a phloroglucinol solution ( $2 \%$ [vol/vol] in $95 \%$ ethanol) for $5 \mathrm{~min}$, followed by immersion in $18 \%$ hydrochloric acid for $5 \mathrm{~min}$, then fixed on slides to be observed and photographed under a fluorescence microscope (SteREO Discovery.V20; Zeiss) immediately. Red color indicated the presence of lignin.

\section{Determination of total lignin content and lignin monomer.}

The total lignin content of stem tissue was determined by the acetyl bromide method as described previously, with minor modifications (Fukushima and Hatfield 2004). Briefly, stem samples (approximately $100 \mathrm{mg}$; the precise weight was recorded) were quickly ground to powder in liquid nitrogen, then successively washed with ethanol, $95 \%$ ethanol, and deionized water to remove soluble substances. After freeze drying, $5 \mathrm{ml}$ of acetyl bromide-glacial acetic acid $(1: 3, \mathrm{vol} / \mathrm{vol})$ was added into each sample and incubated at $70^{\circ} \mathrm{C}$ for $30 \mathrm{~min}$. Then, $0.9 \mathrm{ml}$ of $2 \mathrm{M} \mathrm{NaOH}$ and $3 \mathrm{ml}$ of glacial acetic acid were added, and finally supplied with $1 \mathrm{ml}$ of $7.5 \mathrm{M}$ hydroxylamine hydrochloride solution to terminate the reaction. After centrifugation at 13,000 $\mathrm{rpm}$, the supernatant was collected, and the absorbance at $280 \mathrm{~nm}$ was measured with a NanoDrop ND-2000 spectrophotometer (Thermo Scientific, Wilmington, DE, U.S.A.). Alkali lignin was used as a standard.

The lignin monomer composition of stems was determined for three biological replicates by the thioacidolysis and gaschromatography mass-spectrometry method (Zhao et al. 2010). The lignin monomer composition was expressed as the percentage of dry weight.

\section{Determination of the total content of flavonoids.}

The total flavonoid content was measured following the $\mathrm{AlCl} 3$ colorimetric assay as previously described, with slight modifications (Dewanto et al. 2002). Briefly, at 48 hpi, stems were collected from mock- and $V$. dahliae-inoculated TRV:00 and TRV:Gh4CL30 plants. Stems were ground into powder in liquid nitrogen, and $100 \mathrm{mg}$ of frozen powder was dissolved with $2 \mathrm{ml}$ of extraction solvent ( $80 \%$ methanol). After centrifugation at $13,000 \mathrm{rpm}, 30 \mu \mathrm{l}$ of $5 \% \mathrm{NaNO}_{2}$ (wt/vol) solution was added to the supernatant and incubated at room temperature. After $5 \mathrm{~min}$ of incubation, $60 \mu \mathrm{l}$ of $10 \%$ (wt/vol) $\mathrm{AlCl}_{3}$ solution was added. Samples were well mixed and incubated at room temperature for $5 \mathrm{~min}$. Finally, $0.2 \mathrm{ml}$ of $1 \mathrm{M} \mathrm{NaOH}$ solution was added to each sample. The absorbance at $500 \mathrm{~nm}$ was immediately measured with a NanoDrop ND-2000 spectrophotometer (Thermo Scientific). Rutin was used as a standard.

\section{Extraction and quantification of phytohormone content.}

The extraction and measurement of the endogenous SA and JA were performed as described by Sun et al. (2014), with slight modifications. Approximately $200 \mathrm{mg}$ of fresh stem from TRV: 00 and TRV:Gh4CL3O plants was ground to a fine powder in liquid nitrogen and extracted twice with $2 \mathrm{ml}$ of precooled $80 \%$ methanol overnight in the dark at $4^{\circ} \mathrm{C}$. The supernatant was filtered with a $0.22-\mathrm{mm}$ filter membrane and dried under $\mathrm{N}_{2}$ at room temperature, then dissolved in $0.4 \mathrm{ml}$ of methanol. The JA and SA levels were quantified using the high-performance liquid chromatography-tandem mass spectrometry (LC/MS) system (AB SCIEX Triple Quad 5500) with JA and SA (SigmaAldrich, St. Louis) as the internal standards.

\section{Analyses of the contents of four phenolic compounds.}

To determinate the endogenous concentration of caffeic acid, coumalic acid, ferulic acid, and cinnamic acid, stem samples from mock- and V. dahliae-inoculated TRV:00 and TRV: Gh4CL30 plants 48 hpi were dried in a lyophilizer and ground to a fine powder. The powder $(0.5 \mathrm{~g})$ was extracted twice with $1 \mathrm{ml}$ of $80 \%$ methanol followed by sonication for $1 \mathrm{~h}$ at $60^{\circ} \mathrm{C}$ and extracted at $4^{\circ} \mathrm{C}$ overnight. The samples were centrifuged at $12,000 \mathrm{rpm}$ for $5 \mathrm{~min}$. The supernatant was collected into a fresh tube and dried with nitrogen gas at room temperature to a final volume of approximately $1 \mathrm{ml}$. After filtering through a $0.45-\mu \mathrm{m}$ membrane filter, the extract was analyzed for the concentrations of phenolics on LC/MS according to a previous study (Proestos and Komaitis 2013).

\section{AUTHOR-RECOMMENDED INTERNET RESOURCES}

Novemagic server: https://magic.novogene.com/customer/main\#/login Primer3 Plus: http://primer3plus.com/cgi-bin/dev/primer3plus.cgi

\section{LITERATURE CITED}

Anders, S., and Huber, W. 2010. Differential expression analysis for sequence count data. Genome Biol. 11:R106.

Atallah, Z. K., Bae, J., Jansky, S. H., Rouse, D. I., and Stevenson, W. R. 2007. Multiplex real-time quantitative PCR to detect and quantify Verticillium dahliae colonization in potato lines that differ in response to Verticillium wilt. Phytopathology 97:865-872.

Bhuiyan, N. H., Selvaraj, G., Wei, Y., and King, J. 2009. Gene expression profiling and silencing reveal that monolignol biosynthesis plays a critical role in penetration defence in wheat against powdery mildew invasion. J. Exp. Bot. 60:509-521.

Boerjan, W., Ralph, J., and Baucher, M. 2003. Lignin biosynthesis. Annu. Rev. Plant Biol. 54:519-546.

Boutigny, A. L., Barreau, C., Atanasova-Penichon, V., Verdal-Bonnin, M. N., Pinson-Gadais, L., and Richard-Forget, F. 2009. Ferulic acid, an efficient inhibitor of type B trichothecene biosynthesis and Tri gene expression in Fusarium liquid cultures. Mycol. Res. 113: 746-753.

Cai, Y. F., He, X. H., Mo, J. C., Sun, Q., Yang, J. P., and Liu, J. G. 2009. Molecular research and genetic engineering of resistance to Verticillium wilt in cotton: A review. Afr. J. Biotechnol. 8:7363-7372.

Deketelaere, S., Tyvaert, L., França, S. C., and Höfte, M. 2017. Desirable traits of a good biocontrol agent against Verticillium Wilt. Front. Microbiol. 8:1186.

Dewanto, V., Wu, X., Adom, K. K., and Liu, R. H. 2002. Thermal processing enhances the nutritional value of tomatoes by increasing total antioxidant activity. J. Agric. Food Chem. 50:3010-3014.

Dimkić, I., Ristivojević, P., Janakiev, T., Berić, T., Trifković, J., MilojkovićOpsenica, D., and Stanković, S. 2016. Phenolic profiles and antimicrobial activity of various plant resins as potential botanical sources of Serbian propolis. Ind. Crops Prod. 94:856-871.

Dixon, R. A. 2001. Natural products and plant disease resistance. Nature 411:843-847.

Dixon, R. A., and Steele, C. L. 1999. Flavonoids and isoflavonoids-A gold mine for metabolic engineering. Trends Plant Sci. 4:394-400.

Dong, Q., Magwanga, R. O., Cai, X., Lu, P., Nyangasi Kirungu, J., Zhou, Z., Wang, X., Wang, X., Xu, Y., Hou, Y., Wang, K., Peng, R., Ma, Z., and Liu, F. 2019. RNA-sequencing, physiological and RNAi analyses provide insights into the response mechanism of the $\mathrm{ABC}$-mediated resistance to Verticillium dahliae infection in cotton. Genes (Basel) 10: 110. 
Du, J., Wang, S., He, C., Zhou, B., Ruan, Y. L., and Shou, H. 2017. Identification of regulatory networks and hub genes controlling soybean seed set and size using RNA sequencing analysis. J. Exp. Bot. 68: 1955-1972.

Ehlting, J., Büttner, D., Wang, Q., Douglas, C. J., Somssich, I. E., and Kombrink, E. 1999. Three 4-coumarate:coenzyme A ligases in Arabidopsis thaliana represent two evolutionarily divergent classes in angiosperms. Plant J. 19:9-20.

Ellendorff, U., Fradin, E. F., de Jonge, R., and Thomma, B. P. 2009. RNA silencing is required for Arabidopsis defence against Verticillium wilt disease. J. Exp. Bot. 60:591-602.

Eynck, C., Koopmann, B., Karlovsky, P., and von Tiedemann, A. 2009. Internal resistance in winter oilseed rape inhibits systemic spread of the vascular pathogen Verticillium longisporum. Phytopathology 99: 802-811.

Feng, H., Yang, Y., Sun, S., Li, Y., Zhang, L., Tian, J., Zhu, Q., Feng, Z., Zhu, H., and Sun, J. 2017. Molecular analysis of caffeoyl residues related to pigmentation in green cotton fibers. J. Exp. Bot. 68:4559-4569.

Fradin, E. F., and Thomma, B. P. 2006. Physiology and molecular aspects of Verticillium wilt diseases caused by $V$. dahliae and V. albo-atrum. Mol. Plant Pathol. 7:71-86.

Fukushima, R. S., and Hatfield, R. D. 2004. Comparison of the acetyl bromide spectrophotometric method with other analytical lignin methods for determining lignin concentration in forage samples. J. Agric. Food Chem. 52:3713-3720.

Gallego-Giraldo, L., Jikumaru, Y., Kamiya, Y., Tang, Y., and Dixon, R. A 2011. Selective lignin downregulation leads to constitutive defense response expression in alfalfa (Medicago sativa L.). New Phytol. 190: 627-639.

Gallego-Giraldo, L., Liu, C., Pose-Albacete, S., Pattathil, S., Peralta, A. G., Young, J., Westpheling, J., Hahn, M. G., Rao, X., Knox, J. P., De Meester, B., Boerjan, W., and Dixon, R. A. 2020. ARABIDOPSIS DEHISCENCE ZONE POLYGALACTURONASE 1 (ADPG1) releases latent defense signals in stems with reduced lignin content. Proc. Natl. Acad. Sci. U.S.A. 117:3281-3290.

Gao, S., Yu, H. N., Xu, R. X., Cheng, A. X., and Lou, H. X. 2015. Cloning and functional characterization of a 4-coumarate CoA ligase from liverwort Plagiochasma appendiculatum. Phytochemistry 111:48-58.

Gao, W., Long, L., Zhu, L. F., Xu, L., Gao, W. H., Sun, L. Q., Liu, L. L., and Zhang, X. L. 2013. Proteomic and virus-induced gene silencing (VIGS) Analyses reveal that gossypol, brassinosteroids, and jasmonic acid contribute to the resistance of cotton to Verticillium dahliae. Mol. Cell. Proteomics 12:3690-3703.

Gayoso, C., Pomar, F., Novo-Uzal, E., Merino, F., and de Ilárduya, O. M. 2010. The Ve-mediated resistance response of the tomato to Verticillium dahliae involves $\mathrm{H} 2 \mathrm{O} 2$, peroxidase and lignins and drives PAL gene expression. BMC Plant Biol. 10:232.

Gill, U. S., Uppalapati, S. R., Gallego-Giraldo, L., Ishiga, Y., Dixon, R. A., and Mysore, K. S. 2018. Metabolic flux towards the (iso)flavonoid pathway in lignin modified alfalfa lines induces resistance against Fusarium oxysporum f. sp. medicaginis. Plant Cell Environ. 41: 1997-2007.

Glazebrook, J. 2005. Contrasting mechanisms of defense against biotrophic and necrotrophic pathogens. Annu. Rev. Phytopathol. 43:205-227.

Gui, J., Shen, J., and Li, L. 2011. Functional characterization of evolutionarily divergent 4-coumarate:coenzyme a ligases in rice. Plant Physiol. 157:574-586.

Guo, S., Zuo, Y., Zhang, Y., Wu, C., Su, W., Jin, W., Yu, H., An, Y., and Li, Q. 2017. Large-scale transcriptome comparison of sunflower genes responsive to Verticillium dahliae. BMC Genomics 18:42.

Hollender, C. A., Kang, C., Darwish, O., Geretz, A., Matthews, B. F., Slovin, J., Alkharouf, N., and Liu, Z. 2014. Floral transcriptomes in woodland strawberry uncover developing receptacle and anther gene networks. Plant Physiol. 165:1062-1075.

Hu, Q., Min, L., Yang, X., Jin, S., Zhang, L., Li, Y., Ma, Y., Qi, X., Li, D., Liu, H., Lindsey, K., Zhu, L., and Zhang, X. 2018. Laccase GhLac1 modulates broad-spectrum biotic stress tolerance via manipulating phenylpropanoid pathway and jasmonic acid synthesis. Plant Physiol. 176:1808-1823

Hu, X., Puri, K. D., Gurung, S., Klosterman, S. J., Wallis, C. M., Britton, M., Durbin-Johnson, B., Phinney, B., Salemi, M., Short, D. P. G., and Subbarao, K. V. 2019. Proteome and metabolome analyses reveal differential responses in tomato -Verticillium dahliae-interactions. J. Proteomics 207:103449.

Hurni, S., Scheuermann, D., Krattinger, S. G., Kessel, B., Wicker, T., Herren, G., Fitze, M. N., Breen, J., Presterl, T., Ouzunova, M., and Keller, B. 2015. The maize disease resistance gene Htn1 against northern corn leaf blight encodes a wall-associated receptor-like kinase. Proc. Natl. Acad. Sci. U.S.A. 112:8780-8785.

Kanehisa, M., Araki, M., Goto, S., Hattori, M., Hirakawa, M., Itoh, M. Katayama, T., Kawashima, S., Okuda, S., Tokimatsu, T., and Yamanishi, Y. 2008. KEGG for linking genomes to life and the environment. Nucleic Acids Res. 36:D480-D484.

Klosterman, S. J., Atallah, Z. K., Vallad, G. E., and Subbarao, K. V. 2009. Diversity, pathogenicity, and management of verticillium species. Annu. Rev. Phytopathol. 47:39-62.

Klosterman, S. J., Subbarao, K. V., Kang, S. C., Veronese, P., Gold, S. E., Thomma, B., Chen, Z. H., Henrissat, B., Lee, Y. H., Park, J. S., GarciaPedrajas, M. D., Barbara, D. J., Anchieta, A., de Jonge, R., Santhanam, P., Maruthachalam, K., Atallah, Z., Amyotte, S. G., Paz, Z., Inderbitzin, P., Hayes, R. J., Heiman, D. I., Young, S., Zeng, Q. D., Engels, R., Galagan, J., Cuomo, C. A., Dobinson, K. F., and Ma, L. J. 2011. Comparative genomics yields insights into niche adaptation of plant vascular wilt pathogens. PLoS Pathog. 7:e1002137.

Konig, S., Feussner, K., Kaever, A., Landesfeind, M., Thurow, C., Karlovsky, P., Gatz, C., Polle, A., and Feussner, I. 2014. Soluble phenylpropanoids are involved in the defense response of Arabidopsis against Verticillium longisporum. New Phytol. 202:823-837.

Langfelder, P., and Horvath, S. 2008. WGCNA: An R package for weighted correlation network analysis. BMC Bioinf. 9:559.

Lanoue, A., Burlat, V., Henkes, G. J., Koch, I., Schurr, U., and Röse, U. S. 2010. De novo biosynthesis of defense root exudates in response to Fusarium attack in barley. New Phytol. 185:577-588.

Lavhale, S. G., Kalunke, R. M., and Giri, A. P. 2018. Structural, functional and evolutionary diversity of 4-coumarate-CoA ligase in plants. Planta 248:1063-1078.

Lee, D., and Douglas, C. J. 1996. Two divergent members of a tobacco 4coumarate:coenzyme A ligase (4CL) gene family. cDNA structure, gene inheritance and expression, and properties of recombinant proteins. Plant Physiol. 112:193-205.

Leyva-Pérez, M. O., Jiménez-Ruiz, J., Gómez-Lama Cabanás, C., Valverde-Corredor, A., Barroso, J. B., Luque, F., and Mercado-Blanco, J. 2018. Tolerance of olive (Olea europaea) cv Frantoio to Verticillium dahliae relies on both basal and pathogen-induced differential transcriptomic responses. New Phytol. 217:671-686.

Li, Y., Kim, J. I., Pysh, L., and Chapple, C. 2015. Four isoforms of Arabidopsis 4-coumarate:CoA ligase have overlapping yet distinct roles in phenylpropanoid metabolism. Plant Physiol. 169:2409-2421.

Liu, H., Guo, Z., Gu, F., Ke, S., Sun, D., Dong, S., Liu, W., Huang, M., Xiao, W., Yang, G., Liu, Y., Guo, T., Wang, H., Wang, J., and Chen, Z. 2017. 4-Coumarate-CoA Ligase-Like gene OsAAE3 negatively mediates the rice blast resistance, floret development and lignin biosynthesis. Front. Plant Sci. 7:2041.

Liu, Q., Luo, L., and Zheng, L. 2018. Lignins: Biosynthesis and biological functions in plants. Int. J. Mol. Sci. 19:335.

Liu, Y., Schiff, M., and Dinesh-Kumar, S. P. 2002. Virus-induced gene silencing in tomato. Plant J. 31:777-786.

Livak, K. J., and Schmittgen, T. D. 2001. Analysis of relative gene expression data using real-time quantitative PCR and the $2^{-\Delta \Delta C T}$ Method. Methods 25:402-408.

Miedes, E., Vanholme, R., Boerjan, W., and Molina, A. 2014. The role of the secondary cell wall in plant resistance to pathogens. Front. Plant Sci. $5: 358$.

Mierziak, J., Kostyn, K., and Kulma, A. 2014. Flavonoids as important molecules of plant interactions with the environment. Molecules 19 16240-16265.

Oliveira, M. B., de Andrade, R. V., Grossi-de-Sá, M. F., and Petrofeza, S. 2015. Analysis of genes that are differentially expressed during the Sclerotinia sclerotiorum-Phaseolus vulgaris interaction. Front. Microbiol. 6:1162.

Proestos, C., and Komaitis, M. 2013. Analysis of naturally occurring phenolic compounds in aromatic plants by RP-HPLC coupled to diode array detector (DAD) and GC-MS after silylation. Foods 2:90-99.

Shaban, M., Miao, Y., Ullah, A., Khan, A. Q., Menghwar, H., Khan, A. H., Ahmed, M. M., Tabassum, M. A., and Zhu, L. 2018. Physiological and molecular mechanism of defense in cotton against Verticillium dahliae. Plant Physiol. Biochem. 125:193-204.

Shadle, G. L., Wesley, S. V., Korth, K. L., Chen, F., Lamb, C., and Dixon, R. A. 2003. Phenylpropanoid compounds and disease resistance in transgenic tobacco with altered expression of L-phenylalanine ammonialyase. Phytochemistry 64:153-161.

Sun, L., Zhu, L., Xu, L., Yuan, D., Min, L., and Zhang, X. 2014. Cotton cytochrome P450 CYP82D regulates systemic cell death by modulating the octadecanoid pathway. Nat. Commun. 5:5372. 
Sun, Q., Jiang, H., Zhu, X., Wang, W., He, X., Shi, Y., Yuan, Y., Du, X., and Cai, Y. 2013. Analysis of sea-island cotton and upland cotton in response to Verticillium dahliae infection by RNA sequencing. BMC Genomics 14:852.

Sun, S., Xiong, X.-P., Zhu, Q., Li, Y.-J., and Sun, J. 2019. Transcriptome sequencing and metabolome analysis reveal genes involved in pigmentation of green-colored cotton fibers. Int. J. Mol. Sci. 20:4838.

Sun, S.-C., Xiong, X.-P., Zhang, X.-L., Feng, H.-J., Zhu, Q.-H., Sun, J., and Li, Y.-J. 2020. Characterization of the $G h 4 C L$ gene family reveals a role of Gh4CL7 in drought tolerance. BMC Plant Biol. 20:125.

Tan, M., Cheng, D., Yang, Y., Zhang, G., Qin, M., Chen, J., Chen, Y., and Jiang, M. 2017. Co-expression network analysis of the transcriptomes of rice roots exposed to various cadmium stresses reveals universal cadmium-responsive genes. BMC Plant Biol. 17:194.

Tian, J. S., Zhang, X. Y., Yang, Y. L., Yang, C. X., Xu, S. Z., Zuo, W. Q., Zhang, W. F., Dong, H. Y., Jiu, X. L., Yu, Y. C., and Zhao, Z. 2017. How to reduce cotton fiber damage in the Xinjiang China. Ind. Crops Prod. 109:803-811.

Tonnessen, B. W., Manosalva, P., Lang, J. M., Baraoidan, M., Bordeos, A., Mauleon, R., Oard, J., Hulbert, S., Leung, H., and Leach, J. E. 2015. Rice phenylalanine ammonia-lyase gene OsPAL4 is associated with broad spectrum disease resistance. Plant Mol. Biol. 87:273-286.

Trapnell, C., Williams, B. A., Pertea, G., Mortazavi, A., Kwan, G., van Baren, M. J., Salzberg, S. L., Wold, B. J., and Pachter, L. 2010. Transcript assembly and quantification by RNA-Seq reveals unannotated transcripts and isoform switching during cell differentiation. Nat. Biotechnol. 28:511-515.

Tronchet, M., Balagué, C., Kroj, T., Jouanin, L., and Roby, D. 2010. Cinnamyl alcohol dehydrogenases-C and D, key enzymes in lignin biosynthesis, play an essential role in disease resistance in Arabidopsis. Mol. Plant Pathol. 11:83-92.

Vanholme, R., Demedts, B., Morreel, K., Ralph, J., and Boerjan, W. 2010. Lignin biosynthesis and structure. Plant Physiol. 153:895-905.

Voelker, S. L., Lachenbruch, B., Meinzer, F. C., Jourdes, M., Ki, C., Patten, A. M., Davin, L. B., Lewis, N. G., Tuskan, G. A., Gunter, L., Decker, S. R., Selig, M. J., Sykes, R., Himmel, M. E., Kitin, P., Shevchenko, O., and Strauss, S. H. 2010. Antisense down-regulation of 4CL expression alters lignification, tree growth, and saccharification potential of fieldgrown poplar. Plant Physiol. 154:874-886.

Vogt, T. 2010. Phenylpropanoid biosynthesis. Mol. Plant 3:2-20.

Wang, G. F., and Balint-Kurti, P. J. 2016. Maize homologs of CCoAOMT and HCT, Two key enzymes in lignin biosynthesis, form complexes with the NLR Rp1 protein to modulate the defense response. Plant Physiol. 171:2166-2177

Wang, L., Feng, Z., Wang, X., Wang, X., and Zhang, X. 2010. DEGseq: An $R$ package for identifying differentially expressed genes from RNA-seq data. Bioinformatics 26:136-138.

Wang, M., Tu, L., Yuan, D., Zhu, D., Shen, C., Li, J., Liu, F., Pei, L., Wang, P., Zhao, G., Ye, Z., Huang, H., Yan, F., Ma, Y., Zhang, L., Liu, M., You, J., Yang, Y., Liu, Z., Huang, F., Li, B., Qiu, P., Zhang, Q., Zhu, L., Jin, S., Yang, X., Min, L., Li, G., Chen, L. L., Zheng, H., Lindsey, K., Lin, Z., Udall, J. A., and Zhang, X. 2019. Reference genome sequences of two cultivated allotetraploid cottons, Gossypium hirsutum and Gossypium barbadense. Nat. Genet. 51:224-229.

Wendel, J. F. 1989. New World tetraploid cottons contain Old World cytoplasm. Proc. Natl. Acad. Sci. U.S.A. 86:4132-4136.
Xiong, X. P., Sun, S. C., Li, Y. J., Zhang, X. Y., Sun, J., and Xue, F. 2019. The cotton WRKY transcription factor GhWRKY70 negatively regulates the defense response against Verticillium dahliae. Crop J. 7:393-402.

Xu, L., Zhang, W., He, X., Liu, M., Zhang, K., Shaban, M., Sun, L., Zhu, J., Luo, Y., Yuan, D., Zhang, X., and Zhu, L. 2014. Functional characterization of cotton genes responsive to Verticillium dahliae through bioinformatics and reverse genetics strategies. J. Exp. Bot. 65: 6679-6692

Xu, L., Zhu, L., Tu, L., Liu, L., Yuan, D., Jin, L., Long, L., and Zhang, X. 2011. Lignin metabolism has a central role in the resistance of cotton to the wilt fungus Verticillium dahliae as revealed by RNA-Seq-dependent transcriptional analysis and histochemistry. J. Exp. Bot. 62:5607-5621.

Yang, C. L., Liang, S., Wang, H. Y., Han, L. B., Wang, F. X., Cheng, H. Q., Wu, X. M., Qu, Z. L., Wu, J. H., and Xia, G. X. 2015. Cotton major latex protein 28 functions as a positive regulator of the ethylene responsive factor 6 in defense against Verticillium dahliae. Mol. Plant 8:399-411.

Young, M. D., Wakefield, M. J., Smyth, G. K., and Oshlack, A. 2010. Gene ontology analysis for RNA-seq: Accounting for selection bias. Genome Biol. 11:R14.

Zhang, T., Hu, Y., Jiang, W., Fang, L., Guan, X., Chen, J., Zhang, J., Saski, C. A., Scheffler, B. E., Stelly, D. M., Hulse-Kemp, A. M., Wan, Q., Liu, B., Liu, C., Wang, S., Pan, M., Wang, Y., Wang, D., Ye, W., Chang, L., Zhang, W., Song, Q., Kirkbride, R. C., Chen, X., Dennis, E., Llewellyn, D. J., Peterson, D. G., Thaxton, P., Jones, D. C., Wang, Q., Xu, X., Zhang, H., Wu, H., Zhou, L., Mei, G., Chen, S., Tian, Y., Xiang, D., Li, X., Ding, J., Zuo, Q., Tao, L., Liu, Y., Li, J., Lin, Y., Hui, Y., Cao, Z., Cai, C., Zhu, X., Jiang, Z., Zhou, B., Guo, W., Li, R., and Chen, Z. J. 2015 Sequencing of allotetraploid cotton (Gossypium hirsutum L. acc. TM-1) provides a resource for fiber improvement. Nat. Biotechnol. 33:531-537.

Zhang, W., Zhang, H., Liu, K., Jian, G., Qi, F., and Si, N. 2017. Large-scale identification of Gossypium hirsutum genes associated with Verticillium dahliae by comparative transcriptomic and reverse genetics analysis. PLoS One 12:e0181609.

Zhang, Y., Wang, X., Rong, W., Yang, J., Li, Z., Wu, L., Zhang, G., and Ma, Z. 2017. Histochemical analyses reveal that stronger intrinsic defenses in Gossypium barbadense than in $G$. hirsutum are associated with resistance to Verticillium dahliae. Mol. Plant-Microbe Interact. 30: 984-996.

Zhang, Y., Wang, X. F., Ding, Z. G., Ma, Q., Zhang, G. R., Zhang, S. L., Li, Z. K., Wu, L. Q., Zhang, G. Y., and Ma, Z. Y. 2013. Transcriptome profiling of Gossypium barbadense inoculated with Verticillium dahliae provides a resource for cotton improvement. BMC Genomics 14:637.

Zhang, Y., Wu, L., Wang, X., Chen, B., Zhao, J., Cui, J., Li, Z., Yang, J., Wu, L., Wu, J., Zhang, G., and Ma, Z. 2019. The cotton laccase gene GhLAC15 enhances Verticillium wilt resistance via an increase in defence-induced lignification and lignin components in the cell walls of plants. Mol. Plant Pathol. 20:309-322.

Zhao, Q., Gallego-Giraldo, L., Wang, H., Zeng, Y., Ding, S. Y., Chen, F., and Dixon, R. A. 2010. An NAC transcription factor orchestrates multiple features of cell wall development in Medicago truncatula. Plant J. 63:100-114.

Zhou, X., Jacobs, T. B., Xue, L. J., Harding, S. A., and Tsai, C. J. 2015. Exploiting SNPs for biallelic CRISPR mutations in the outcrossing woody perennial Populus reveals 4-coumarate:CoA ligase specificity and redundancy. New Phytol. 208:298-301. 